\title{
Combinatorial Groupoids, Cubical Complexes, and the Lovász Conjecture
}

\author{
Rade T. Živaljević
}

Received: 13 May 2007 / Revised: 13 January 2008 /

Published online: 5 February 2008

(C) Springer Science+Business Media, LLC 2008

\begin{abstract}
This paper lays the foundation for a theory of combinatorial groupoids that allows us to use concepts like "holonomy", "parallel transport", "bundles", "combinatorial curvature", etc. in the context of simplicial (polyhedral) complexes, posets, graphs, polytopes and other combinatorial objects. We introduce a new, holonomy-type invariant for cubical complexes, leading to a combinatorial "Theorema Egregium" for cubical complexes that are non-embeddable into cubical lattices. Parallel transport of Hom-complexes and maps is used as a tool to extend BabsonKozlov-Lovász graph coloring results to more general statements about nondegenerate maps (colorings) of simplicial complexes and graphs.
\end{abstract}

Keywords Combinatorial groupoids · Lovász conjecture · Cubical complexes · Discrete differential geometry

\section{Introduction}

There has been new and encouraging evidence that the language and methods of the theory of groupoids, after being successfully applied in other major mathematical fields, offer new insights and perspectives for applications in combinatorics and discrete and computational geometry.

The groupoids (groups of projectivities) have recently appeared in geometric combinatorics in the work of M. Joswig [28]; see also a related paper with Izmestiev [27] and the references to these papers, where they have been applied to toric manifolds, branched coverings over $S^{3}$, colorings of simple polytopes etc.

The author was supported by grants 144014 and 144026 of the Serbian Ministry of Science and Technology.

R.T. Živaljević (凶)

Mathematical Institute SANU, Knez Mihailova 35/1, p.f. 367, 11001 Belgrade, Serbia

e-mail: rade@mi.sanu.ac.yu 
The purpose of this paper is to show that these developments should not be seen as isolated examples. Quite the opposite, they serve as a motivation for further extensions and generalizations. Our first application shows how a cubical analogue of Joswig's groupoid provides new insight about cubical complexes that are nonembeddable into cubical lattices (a question related to a problem of S.P. Novikov which arose in connection with the three-dimensional Ising model) [13, 39]. The second application leads to a new proof and a generalization, from graphs to simplicial complexes, of a recent resolution of the Lovász conjecture by Babson and Kozlov [4, 34].

Combinatorial groupoids have appeared (implicitly) in other contemporary combinatorial constructions and applications, see Babson et al. [1], recent work of Barcelo et al. on Atkin-homotopy [5, 6] etc. Bolker, Guillemin, and Holm [10] defined connections on graphs and, guided by the analogies between graphs and manifolds, applied them to the parallel redrawing problem. Novikov [40]—-see also Novikov and Dynnikov [16] — studied discrete connections on triangulated manifolds as part of a general program of discretizing differential geometric concepts, finding discrete analogues of complete integrable systems etc.

One of the central objectives of our paper is to advocate a systematic use of the language and the technique of groupoids, and to propose their wider recognition as a valuable tool for geometric and algebraic combinatorics.

\subsection{The Unifying Theme}

Our point of departure is an observation that different problems-including the question regarding the existence of an embedding $f: M \hookrightarrow N$ of Riemann manifolds, the existence and classification of embeddings (immersions) of cubical surfaces into hypercubes or cubical lattices, the existence of a coloring of a graph $G=\left(V_{G}, E_{G}\right)$ with a prescribed number of colors etc. — can all be approached from a similar point of view.

The unifying theme and a single point of view is provided by the concept of a groupoid. In each of the listed cases there is a groupoid naturally associated to an object of the given category. For each of these groupoids there is an associated "parallel transport", holonomy groups and other related invariants which serve as obstructions for the existence of morphisms (see Fig. 1).

If $M=(M, g)$ is a Riemann manifold, the associated groupoid $\mathcal{G}=\mathcal{G}_{M}$ has $M$ as the set of objects while the morphism set $\mathcal{G}(x, y)$ consists of all linear isomorphisms $a: T_{x} M \rightarrow T_{y} M$ arising from a parallel transport along piece-wise smooth curves from $x$ to $y$. One of the manifestations of Gauss's "Theorema Egregium" is that the associated holonomy group is a metric invariant, consequently an isometry depicted in Fig. 1a is not possible.

In a similar fashion a cubical "Theorema Egregium" (Theorem 3.7) creates an obstruction to embedding a cubical complex into a hypercube or a cubical lattice. As a consequence the cubulation (quadrangulation) of an annulus depicted in Fig. 1b does not admit a cubical embedding into a hypercube; for the same formal reason (incompatible holonomy) a spherical cap cannot be isometrically represented in the plane. 
(a)

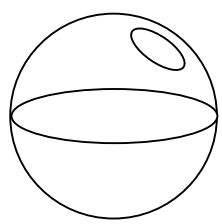

(b)

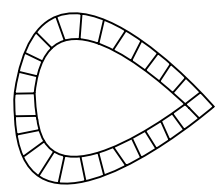

(c)

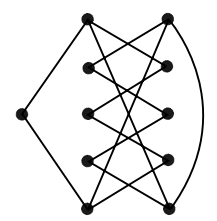

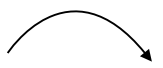

isometry?
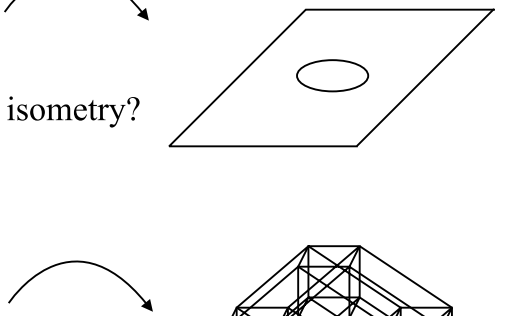

cubical embedding?
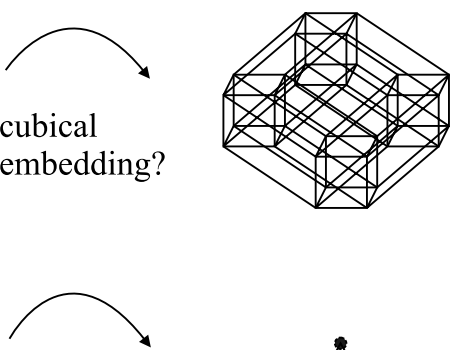

coloring?

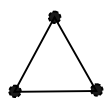

Fig. 1 What is a common point of view?

Perhaps it comes as a surprise that the graph-coloring problem, Fig. 1c, can also be approached from a similar point of view. An analysis of holonomies ("parallel transport") of diagrams of Hom-complexes of graphs (simplicial complexes) over the associated Joswig groupoid eventually leads to a general result (Theorem 4.21) which includes the "odd" case of Babson-Kozlov-Lovász coloring theorem as a special case.

\subsection{An Overview of the Paper}

Our objective is to lay a foundation for a program of associating groupoids to posets, graphs, complexes, arrangements, configurations and other combinatorial structures. This "geometrization of combinatorics" should provide useful guiding principles and transparent geometric language so that we can introduce concepts such as combinatorial holonomy, discrete parallel transport, combinatorial curvature, combinatorial bundles etc. The first steps in this direction are made in Sect. 2.

Section 3, devoted to cubical complexes, is an elaboration of the theme depicted in Fig. 1b. A holonomy $\mathbb{Z}_{2}$-valued invariant $I(K)$ of a cubical complex is introduced. This leads to a "combinatorial curvature" $C C(K)$ and associated "Cubical Theorema Egregium" (Theorem 3.7) which provides necessary conditions for the existence of an embedding/immersion $K \hookrightarrow L$ of cubical complexes.

Section 4, formally linked to Fig. 1c, introduces groupoids to the problem of coloring graphs and complexes. The focus is on the Lovász Hom-conjecture and its ramifications. We introduce parallel transport of graph-complexes and more general Hom-complexes over graphs/complexes, and we establish fundamental invariance of 
homotopy types of maps in Propositions 4.3 and 4.16. This ultimately leads to general results about coloring of graphs and simplicial complexes (Theorems 4.21 and 4.24, Corollary 4.23).

\section{Groupoids}

It is, or should be, well known that the concept of a group is sometimes not sufficient to deal with the concept of symmetry in general. Groupoids, understood as groups with "space-like properties", often allow us to handle objects which exhibit what is clearly recognized as symmetry although they admit no global automorphism whatsoever. Unlike groups, groupoids are capable of describing reversible processes which can pass through a number of states. For example, according to Connes [15], Heisenberg discovered quantum mechanics by considering the groupoid of quantum transitions rather than the group of symmetry.

By definition a groupoid is a small category $\mathcal{C}=(\operatorname{Ob}(\mathcal{C}), \operatorname{Mor}(\mathcal{C}))$ such that each morphism $\alpha \in \operatorname{Mor}(\mathcal{C})$ is an isomorphism. We follow the usual terminology and notation $[11,12,24,46]$. Perhaps the only exception is that we sometimes call the vertex (isotropy) group $\Pi(\mathcal{C}, x):=\mathcal{C}(x, x)$ the holonomy group of $\mathcal{C}$ at $x \in O b(\mathcal{C})$. If the groupoid $\mathcal{C}$ is connected all vertex (holonomy) groups $\Pi(\mathcal{C}, x)$ are isomorphic and their isomorphism type is denoted by $\Pi(\mathcal{C})$.

\subsection{Generalities About "Bundles" and "Parallel Transport"}

The notion of groupoid is a common generalization of the concepts of space and group, i.e. the theory of groupoids allows us to treat spaces, groups and objects associated to them from the same point of view. A common generalization for the concept of a bundle $Y$ over $X$ and a $G$-space $Y$ is a $\mathcal{C}$-space or more formally a diagram over the groupoid $\mathcal{C}$ defined as a functor $F: \mathcal{C} \rightarrow$ Top.

In order to preserve the original intuition we use a geometric language which resembles the usual concepts as much as possible. In particular we clarify what is in this paper meant by a $\mathcal{C}$-parallel transport on a "bundle" of spaces over a set $S$.

A (naive) "bundle" is a map $\phi: X \rightarrow S$. We assume that $S$ is a set and that $X(i):=$ $\phi^{-1}(i)$ is a topological space, so a bundle is just a collection of spaces (fibres) $X(i)$ parameterized by $S$. If all spaces $X(i)$ are homeomorphic to a fixed "model" space, this space is referred to as the fiber of the bundle $\phi$.

Suppose that $\mathcal{C}$ is a groupoid on $S$ as the set of objects. In other words $\mathcal{C}=$ $(\operatorname{Ob}(\mathcal{C}), \operatorname{Mor}(\mathcal{C}))$ is a small category where $\operatorname{Ob}(\mathcal{C})=S$, such that all morphisms $\alpha \in \operatorname{Mor}(\mathcal{C})$ are invertible.

A "connection" or "parallel transport" on the bundle $\mathcal{X}=\{X(i)\}_{i \in S}$ is a functor (diagram) $\mathcal{P}: \mathcal{C} \rightarrow$ Top such that $X(i)=\mathcal{P}(i)$ for each $i \in S$.

Informally speaking, the groupoid $\mathcal{C}$ provides a "road map" on $S$, while the functor $\mathcal{P}$ defines the associated transport from one fibre to another.

Sometimes it is convenient to view the bundle $\mathcal{X}=\{X(i)\}_{i \in S}$ as a map $\mathcal{X}: S \rightarrow$ Top. Then defining a "connection" on this bundle is equivalent to enriching the map $\mathcal{X}$ to a functor $\mathcal{P}: \mathcal{C} \rightarrow$ Top. 


\subsection{Combinatorial Groupoids}

The following definition introduces the first in a series of combinatorial groupoids.

Definition 2.1 Suppose that $(P, \leq)$ is a (not necessarily finite) poset. Suppose that $\Sigma$ and $\Delta$ are two families of subposets of $P$. Choose $\sigma_{1}, \sigma_{2} \in \Sigma$. If for some $\delta \in \Delta$ both $\delta \subset \sigma_{1}$ and $\delta \subset \sigma_{2}$, then the posets $\sigma_{1}, \sigma_{2}$ are called $\delta$-adjacent, or just adjacent if $\delta$ is not specified. Define $\mathcal{C}=(\operatorname{Ob}(\mathcal{C}), \operatorname{Mor}(\mathcal{C}))$ as a small category over $\operatorname{Ob}(\mathcal{C})=\Sigma$ as the set of objects as follows. For two $\delta$-adjacent objects $\sigma_{1}$ and $\sigma_{2}$, an elementary morphism $\alpha \in \mathcal{C}\left(\sigma_{1}, \sigma_{2}\right)$ is an isomorphism $\alpha: \sigma_{1} \rightarrow \sigma_{2}$ of posets which leaves $\delta$ point-wise fixed. A morphism $\mathfrak{p} \in \mathcal{C}\left(\sigma_{0}, \sigma_{m}\right)$ from $\sigma_{0}$ to $\sigma_{m}$ is an isomorphism of posets $\sigma_{0}$ and $\sigma_{m}$ which can be expressed as a composition of elementary morphisms.

Given two adjacent objects $\sigma_{1}$ and $\sigma_{2}$, an elementary morphism $\alpha \in \mathcal{C}\left(\sigma_{1}, \sigma_{2}\right)$ may not exist at all, or if it exists it may not be unique. In case it exists and is unique it will be frequently denoted by $\overrightarrow{\sigma_{1} \sigma_{2}}$ and sometimes referred to as a "flip" from $\sigma_{1}$ to $\sigma_{2}$. In this case a morphism $\mathfrak{p} \in \mathcal{C}\left(\sigma_{0}, \sigma_{m}\right)$ is by definition a composition of flips

$$
\mathfrak{p}=\overrightarrow{\sigma_{0} \sigma_{1}} * \overrightarrow{\sigma_{1} \sigma_{2}} * \cdots * \overrightarrow{\sigma_{n-1}}+
$$

Caveat Here we adopt a useful convention that $(x)(f * g)=(g \circ f)(x)$ for each two composable maps $f$ and $g$. The notation $f * g$ is often given priority over the usual $g \circ f$ if we want to emphasize that the functions act on the points from the right, that is, if the arrows in the associated formulas point from left to the right.

Remark 2.2 Definition 2.1 can be restated in a much more general form. For example the ambient poset $P$ can be replaced by a small category $\mathcal{G}, \Sigma$ and $\Delta$ by families of subobjects of $\mathcal{G}$, elementary morphisms are defined as commutative diagrams etc. However, our objective in this paper is not to explore all the possibilities. Instead we create an "ecological niche" for combinatorial groupoids which may be populated by new examples and variations as the theory develops.

Suppose that $P$ is a ranked poset of depth $n$ with the associated rank function $r: P \rightarrow[n]$. Let $\mathcal{E}=\mathcal{E}_{P}$ be the $\mathcal{C}$-groupoid described in Definition 2.1 associated to the families $\Sigma:=\left\{P_{\leq x} \mid r(x)=n\right\}$ and $\Delta:=\left\{P_{\leq y} \mid r(y)=n-1\right\}$. It is clear that other "rank selected" groupoids can be similarly defined.

The definitions of groupoids $\mathcal{C}$ and $\mathcal{E}$ are easily extended from posets to simplicial, polyhedral or other classes of cell complexes. If $K$ is a complex and $P:=P_{K}$ the associated face poset, then $\mathcal{C}_{K}$ and $\mathcal{E}_{K}=\mathcal{E}(K)$ are groupoids associated to the poset $P_{K}$. We will usually drop the subscript whenever it is clear from the context what is the ambient poset $P$ or complex $K$.

Example 2.3 Suppose that $K$ is a pure, $d$-dimensional simplicial complex. Let $\mathcal{E}(K)$ be the associated $\mathcal{E}$-groupoid corresponding to ranks $d$ and $d-1$. Then the groups of projectivities $\Pi(K, \sigma)$, introduced by Joswig in [28], are nothing but the holonomy groups of the groupoid $\mathcal{E}(K)$. For this reason the groupoid $\mathcal{E}(K)$ is in the sequel often referred to as Joswig's groupoid and denoted by $\mathcal{J}(K) \cdot \mathcal{J}(K)$ is connected as a groupoid if and only if $K$ is "strongly connected" in the sense of [28]. 
A simplicial map of simplicial complexes is nondegenerate if it is $1-1$ on simplices. The following definition extends this concept to the case of posets.

Definition 2.4 A monotone map of posets $f: P \rightarrow Q$ is non-degenerate if the restriction of $f$ on $P_{\leq x}$ induces an isomorphism of posets $P_{\leq x}$ and $Q_{\leq f(x)}$ for each element $x \in P$. Similarly, a map of simplicial, cubical or more general cell complexes is nondegenerate if the associated map of posets is nondegenerate. In this case we say that $P$ is mappable to $Q$ while a nondegenerate map $f: P \rightarrow Q$ is often referred to as a combinatorial immersion from $P$ to $Q$.

Example 2.5 A graph homomorphism [34] $f: G_{1} \rightarrow G_{2}$ can be defined as a nondegenerate map of associated one-dimensional cell complexes. An $n$-coloring of a graph $G$ is a non-degenerate map (graph homomorphism) $f: G \rightarrow K_{n}$ where $K_{n}$ is a complete graph on $n$ vertices.

Proposition 2.6 Suppose that $P$ and $Q$ are ranked posets of depth $n$ and let $f$ : $P \rightarrow Q$ be a nondegenerate map. Then there is an induced map (functor) $F$ : $\mathcal{E}_{P} \rightarrow \mathcal{E}_{Q}$ of the associated $\mathcal{E}$-groupoids. Moreover, $F$ induces an inclusion map $\Pi\left(\mathcal{E}_{P}, \mathfrak{p}\right) \hookrightarrow \Pi\left(\mathcal{E}_{Q}, F(\mathfrak{p})\right)$ of the associated holonomy groups.

\section{Cubical Complexes}

The following two problems serve as a motivation for studying nondegenerate morphisms between cubical complexes, the case of embeddings being of special importance.

$\mathrm{P}_{1}$ (S.P. Novikov) Characterize $k$-dimensional complexes that admit a (cubical) embedding, or more generally a "combinatorial immersion", into the standard cubical lattice of $\mathbb{R}^{d}$ for some $d[13,39]$.

$\mathrm{P}_{2}$ (N. Habegger) Suppose we have two cubulations of the same manifold. Are they related by the bubble moves [31]?

The first question was according to [13] motivated by a problem from statistical physics (three-dimensional Ising model). The second question initiated the study of "bubble moves" on cubical subdivisions (cubulations) of manifolds and complexes which are analogues of stellar operations in simplicial category. For these and related questions about cubical complexes the reader is referred to [2, 7, 17-22, 30, 43] and the references in these papers.

Recall that a cell complex $K$ is cubical if it is a regular $C W$-complex such that the associated face poset $P_{K}$ is cubical in the sense of the following definition.

Definition 3.1 $P$ is a cubical poset if:

(a) For each $x \in P$, the subposet $P_{\leq x}$ is isomorphic to the face poset of some cube $I^{q}$.

(b) $P$ is a semilattice in the sense that if a pair $x, y \in P$ is bounded from above then it has the least upper bound. 
If a space $X$ comes equipped with a standard cubulation, clear from the context, this cubical complex is denoted by $\{X\}$, the associated $k$-skeleton is denoted by $\{X\}_{(k)}$ etc. For example, $\left\{I^{d}\right\}_{(k)}$ is the $k$-skeleton of the standard cubulation of the $d$-cube.

The group $B C_{k}$ of all symmetries of a $k$-cube is isomorphic to the group of all signed, permutation $(k \times k)$-matrices. Its subgroup of all matrices with even number of $(-1)$-entries is denoted by $B C_{k}^{\text {even }}$.

Theorem 3.2 Suppose that $K$ is a $k$-dimensional cubical complex which is embeddable/mappable to $\left\{\mathbb{R}^{n}\right\}_{(k)}$, the k-dimensional skeleton of the standard cubical decomposition $\left\{\mathbb{R}^{n}\right\}=\left\{\mathbb{R}^{1}\right\}^{n}$ of $\mathbb{R}^{n}$. Then $\Pi(K, \sigma) \subset B C_{k}^{\text {even }}$ for each cube $\sigma \in K$. Moreover, if $\sigma \in L \subset K$ where $L \cong\left\{I^{k+1}\right\}_{(k)}$ is a subcomplex of $K$ isomorphic to the $k$-skeleton of the $(k+1)$-dimensional cube $\left\{I^{k+1}\right\}$, then

$$
\Pi(K, \sigma) \cong B C_{k}^{e v e n}
$$

Proof The finiteness of the holonomy group $\Pi(K, \sigma)$ allows us to assume that the complex $K$ is also finite, hence embeddable/mappable to $\left\{[0, v]^{n}\right\}_{(k)}$ for some integer $v \geq 1$. The case $k=1$ of the theorem is a consequence of the simple fact that each closed edge-path in $\left\{\mathbb{R}^{n}\right\}$ is of even length, so let us assume that $k \geq 2$.

Each finite cubical subcomplex of $\left\{\mathbb{R}^{n}\right\}_{(k)}$ is embeddable in a cube $\left\{I^{d}\right\}_{(k)}$ for sufficiently large $d$. Indeed, the 1-complex $\left\{I^{d}\right\}_{(1)}$ is a Hamiltonian graph with $2^{d}$-vertices which implies that the "chain" $\{[a, b]\} \subset\left\{\mathbb{R}^{1}\right\}$ is embeddable in the 1 -skeleton of the cube $\left\{I^{d}\right\}$ if $b-a \leq 2^{d}-1$. Since $\left\{I^{d_{1}}\right\} \times\left\{I^{d_{2}}\right\} \cong\left\{I^{d_{1}+d_{2}}\right\}$, we observe that $\left\{[a, b]^{n}\right\}$ is embeddable in $\left\{I^{d}\right\}$ for some $d$, hence the same holds for their $k$-skeletons.

In light of Proposition 2.6, the holonomy group $\Pi(K, \sigma)$ is a subgroup of $\Pi\left(\left\{I^{d}\right\}_{(k)}\right)$ for some $d>k$ so it is sufficient to establish the result for the complex $K=\left\{I^{d}\right\}_{(k)}$ where $d>k \geq 2$.

As a preliminary step let us recall some basic facts about the $d$-cube $I^{d}$. Assume that $I^{d} \subset \mathbb{R}^{d}$ and let $\left[e_{1}, e_{2}, \ldots, e_{d}\right]$ be the standard orthonormal frame in $\mathbb{R}^{d}$. Let $I_{x_{i}=0}^{d}=\left\{\bar{x} \in I^{d} \mid x_{i}=0\right\}$, respectively $I_{x_{i}=1}^{d}=\left\{\bar{x} \in I^{d} \mid x_{i}=1\right\}$, be the front, respectively back face of $I^{d}$ in the direction of the basic vector $e_{i}$. Given a face $\sigma \in\left\{I^{d}\right\}$, let

$$
I_{0}(\sigma):=\left\{i \in[d] \mid \sigma \subset I_{x_{i}=0}^{d}\right\} \quad \text { and } \quad I_{1}(\sigma):=\left\{i \in[d] \mid \sigma \subset I_{x_{i}=1}^{d}\right\} .
$$

By definition $\sigma=\left\{\bar{x} \in I^{d} \mid x_{i}=0\right.$ and $x_{j}=1$ for each $i \in I_{0}(\sigma)$ and $\left.j \in I_{1}(\sigma)\right\}$. Let $I(\sigma):=[d] \backslash\left(I_{0}(\sigma) \cup I_{1}(\sigma)\right)$. A cube $\tau \in\left\{I^{d}\right\}$ is a front face, respectively, back face of $\sigma$, if for some $i \in I(\sigma), \tau=\sigma \cap I_{x_{i}=0}^{d}$, respectively $\tau=\sigma \cap I_{x_{i}=1}^{d}$.

Suppose that $\sigma_{0}$ and $\sigma_{1}$ are two adjacent $k$-dimensional faces in $\left\{I^{d}\right\}$ such that $\tau:=\sigma_{0} \cap \sigma_{1}$ is their common $(k-1)$-dimensional face. Let $\overrightarrow{\sigma_{0} \sigma_{1}} \in \mathcal{E}\left(\sigma_{0}, \sigma_{1}\right)$ be the associated element in the combinatorial groupoid of $\left\{I^{d}\right\}_{(k)}$ (see Sect. 2.2). Recall that $\overrightarrow{\sigma_{0} \sigma_{1}}: \sigma_{0} \rightarrow \sigma_{1}$ is the isomorphism of cell complexes (posets) which keeps the subcomplex $\{\tau\}$ fixed. Since $\sigma_{0}$ and $\sigma_{1}$ are faces of a regular cube, there is a unique isometry $R_{\sigma_{0} \sigma_{1}}: \sigma_{0} \rightarrow \sigma_{1}$ of faces $\sigma_{0}$ and $\sigma_{1}$ which keeps the common face $\tau$ pointwise fixed. Let $T\left(\sigma_{0}\right)$ and $T\left(\sigma_{1}\right)$ be the $k$-dimensional linear subspaces in $\mathbb{R}^{d}$ tangent (that is parallel) to $\sigma_{0}$ and $\sigma_{1}$, respectively, and let $r_{\sigma_{0} \sigma_{1}}: T\left(\sigma_{0}\right) \rightarrow T\left(\sigma_{1}\right)$ be the 


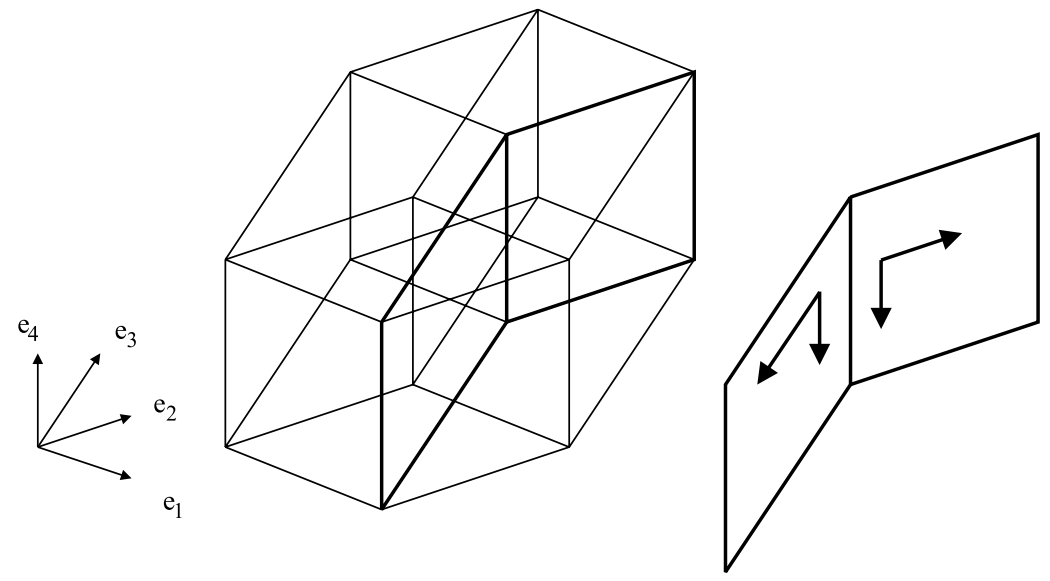

Fig. 2 The effect of a "flip" on the sign characteristic

corresponding isometry associated to $R_{\sigma_{0} \sigma_{1}}$. The introduction of these maps allows us to pass from the combinatorial groupoid $\mathcal{E}=\mathcal{E}\left(\left\{I^{d}\right\}_{(k)}\right)$ to the isomorphic groupoid $\mathcal{E}_{\text {iso }}=\mathcal{E}_{\text {iso }}\left(\left\{I^{d}\right\}_{(k)}\right)$ of isometries of $k$-faces in $I^{d}$.

The following lemma is the essential step in describing the action of the groupoid $\mathcal{E}_{i s o}$ on the set of admissible frames on $k$-faces of the cube $I^{d}$. By definition a frame $f_{a}=\left[a_{1}, a_{2}, \ldots, a_{k}\right]$ is admissible for a face $\sigma$ if $a_{i}=\epsilon_{i} e_{v_{i}}$ for each $i=1, \ldots, k$ where $\epsilon_{i} \in\{-1,+1\}$ and $\left(v_{1}, v_{2}, \ldots, v_{k}\right)$ is some permutation of the set $I(\sigma)$. The sign characteristic $\operatorname{sc}\left(f_{a}\right)$ of an admissible frame $f_{a}$ is the number of negative signs in the sequence $\epsilon_{1}, \epsilon_{2}, \ldots, \epsilon_{k}$.

Lemma The linear isometry $r_{\sigma_{0} \sigma_{1}}: T\left(\sigma_{0}\right) \rightarrow T\left(\sigma_{1}\right)$ maps a frame $f_{a}=\left[a_{1}, \ldots a_{k}\right]$, admissible for $\sigma_{0}$, to a frame $f_{b}=\left[b_{1}, \ldots, b_{k}\right]$ admissible for the face $\sigma_{1}$ where $b_{j}=$ $\eta_{j} e_{\mu_{j}}$ for each $j$, such that $\eta_{j} \in\{-1,+1\}$ and $\left(\mu_{1}, \mu_{2}, \ldots, \mu_{k}\right)$ is a permutation of $I\left(\sigma_{1}\right)$. Moreover, $\operatorname{sc}\left(f_{a}\right)=s c\left(f_{b}\right)$ if $\tau$ is either a front face for both $\sigma_{0}$ and $\sigma_{1}$ or a back face for both $\sigma_{0}$ and $\sigma_{1}$. In the opposite case the parity of the sign characteristic changes, more precisely $s c\left(f_{b}\right)=s c\left(f_{a}\right) \pm 1$.

The proof of the lemma is shown in Fig. 2, which illustrates the case when the sign characteristic of a frame is affected by the flip from $\sigma_{0}$ to $\sigma_{1}$.

Suppose that $\sigma_{0}, \sigma_{1}, \ldots, \sigma_{m}=\sigma_{0}$ is a sequence of adjacent $k$-faces in $\left\{I^{d}\right\}_{(k)}$ and let $\mathfrak{p}=\overrightarrow{\sigma_{0} \sigma_{1}} * \overrightarrow{\sigma_{1} \sigma_{2}} * \cdots * \overrightarrow{\sigma_{m-1} \sigma_{0}}$ be the associated element in $\Pi\left(\left\{I^{d}\right\}_{(k)}, \sigma_{0}\right)$. Let $R$ : $\sigma_{0} \rightarrow \sigma_{0}$ be the isometry associated to $\mathfrak{p}$ and $r: T\left(\sigma_{0}\right) \rightarrow T\left(\sigma_{0}\right)$ the corresponding linear isometry.

Assume that $I\left(\sigma_{0}\right)=\left\{i_{1}, i_{2}, \ldots, i_{k}\right\}$ which implies that $\left[e_{i_{1}}, e_{i_{2}}, \ldots, e_{i_{k}}\right]$ is the canonical admissible frame for $\sigma_{0}$. By a successive application the lemma on pairs $\left(\sigma_{j}, \sigma_{j+1}\right)$, we observe that $r\left[e_{i_{1}}, e_{i_{2}}, \ldots, e_{i_{k}}\right]=\left[\epsilon_{1} e_{\nu_{1}}, \epsilon_{2} e_{\nu_{2}}, \ldots, \epsilon_{k} e_{\nu_{k}}\right]$ where $\left(v_{1}, v_{2}, \ldots, v_{k}\right)$ is a permutation of the set $I\left(\sigma_{0}\right)$ such that the number of negative signs in the sequence $\left(\epsilon_{1}, \epsilon_{2}, \ldots, \epsilon_{k}\right)$ must be even. From here we deduce that $r \in B C_{k}^{\text {even }}$. 
Fig. 3 Cubical complex non-embeddable into a cubical lattice
(A)

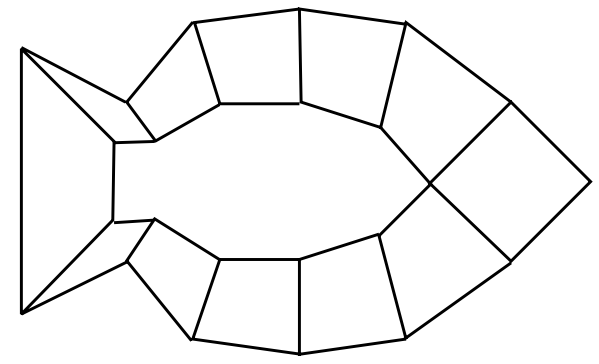

(B)

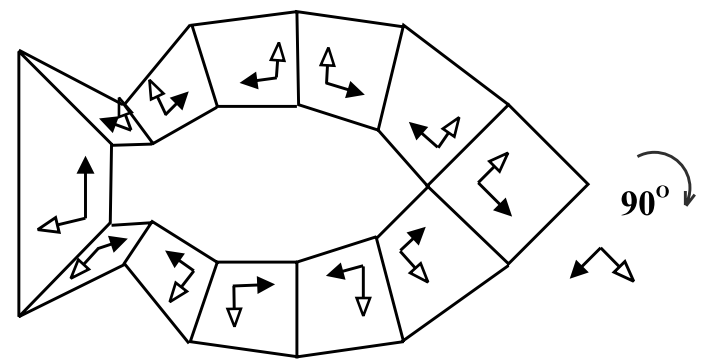

One easily proves by inspection that $\Pi\left(\left\{I^{3}\right\}_{(2)}\right) \cong B C_{3}^{\text {even }} \cong \mathbb{Z}_{2} \oplus \mathbb{Z}_{2}$. This in turn provides a key step for the proof that $\Pi\left(\left\{I^{k+1}\right\}_{(k)}\right) \cong B C_{k}^{e v e n}$ which completes the proof of the second part of the theorem.

Corollary 3.3 The complex $K$ depicted in Fig. 3 is not embeddable (mappable) to a cubical lattice (hypercube) of any dimension. Indeed,

$$
\left[\begin{array}{cc}
0 & 1 \\
-1 & 0
\end{array}\right] \in \Pi(K, \sigma)
$$

while by Theorem 3.2 only signed permutation matrices with an even number of $(-1)$ entries can arise as holonomies of subcomplexes of cubical lattices!

Theorem 3.2 and Corollary 3.3 serve as a motivation for introducing an invariant $I(K) \in \mathbb{Z}_{2}$ of a cubical complex $K$ and an associated "combinatorial curvature" $C C(K)$ of $K$. Both invariants, especially the invariant $C C(K)$, can be used for testing if there exists an embedding (nondegenerate mapping) $f: K \rightarrow L$ of cubical complexes $K$ and $L$. The $\mathbb{Z}_{2}$-characteristics $I(K)$ is cruder than $C C(K)$, however, it possesses an additional property that it is invariant with respect to bubble moves, Proposition 3.8.

Definition 3.4 Suppose that $K$ is a $k$-dimensional cubical complex and let $\Pi(K, \sigma)$ be its combinatorial holonomy group based at $\sigma \in K$. By definition let $I(K)=0$ if $\Pi(K, \sigma) \subset B C_{k}^{\text {even }}$ for all $\sigma$, and $I(K)=1$ in the opposite case.

Definition 3.5 Given a $k$-dimensional cubical complex, let $Z(K):=\inf \left\{f_{k}(W) \mid\right.$ $W \subset K$ and $I(W)=1\}$ where $f_{j}(K)$ is the number of $j$-dimensional cells (cubes) 
in $K$. In particular $Z(K)=+\infty$ if $I(K)=0$. The combinatorial curvature of $K$ is the number $C C(K):=1 / Z(K)$ which is by definition 0 if $I(K)=0$.

Remark 3.6 Both $I(K)$ and $C C(K)$ are global invariants of the cubical $k$-complex $K$. One can introduce a local invariant $Z(K, \sigma)$ as the minimum length $m$ of a closed chain $\sigma=\sigma_{0}, \ldots, \sigma_{m}=\sigma$ of adjacent cubes in $K$ such that

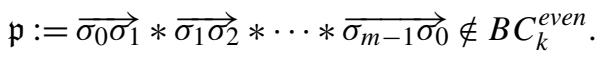

Then $C C(K, \sigma):=1 / Z(K, \sigma)$ is the combinatorial curvature of $K$ at $\sigma$ and $C C(K)=\sup _{\sigma \in K^{(k)}}\{C(K, \sigma)\}$. This not only provides a "correct" answer in the case of hypercubes (cubical lattices) but resembles one of the usual definitions of the curvature as the (limit) quotient of locally defined quantities.

The following theorem summarizes the monotonicity properties of invariants $Z(K)$ and $C C(K)$ in the form suitable for immediate applications.

Theorem 3.7 (Cubical "Theorema Egregium") If there exists an embedding or more generally a nondegenerate mapping (cubical immersion) $f: K \rightarrow L$ of $k$ dimensional cubical complexes $K$ and $L$, then

$$
Z(K) \geq Z(L) \quad \text { or equivalently } \quad C C(K) \leq C C(L)
$$

In other words the "curvature" of $K$ must not exceed the "curvature" of $L$ if $K$ is to be embedded in $L$.

The following result shows the relevance of the invariant $I(K)$ for the problem of Habegger (problem $P_{2}$ ). It says that $I(K)$ is invariant with respect to cubical modifications known as "bubble moves" [20, 22].

Proposition 3.8 Suppose that $K$ is a $k$-dimensional cubical complex. Then $I(K)$ is invariant with respect to bubble moves, that is $I(K)=I\left(K^{\prime}\right)$ where $K^{\prime}$ is the complex obtained from $K$ by a sequence of bubble moves.

Proof Suppose that $K^{\prime}$ is obtained from $K$ by a bubble move. By definition this means that $K^{\prime}$ can be obtained from $K$ by excising $B$ from $K$ and replacing it by $B^{\prime}$ where $B$ and $B^{\prime}$ are complementary balls in the boundary of the $(k+1)$ cube, see Fig. 4. Each cubical path $\mathfrak{p}$ in $K$ which enters the ball $B \subset K$ can be modified to a cubical path $\mathfrak{p}^{\prime}$ by replacing each cubical fragment of $\mathfrak{p}$ in $B$ by a corresponding fragment in $B^{\prime}$. Then the equality $I(K)=I\left(K^{\prime}\right)$ follows from $I\left(\left\{I^{k+1}\right\}_{(k)}\right)=0$.

Corollary 3.9 If $I(K)=1$ and $I(L)=0$, then there does not exist an embedding (combinatorial immersion) from $K$ to $L$, even after $K$ and $L$ are modified by some bubble moves. 

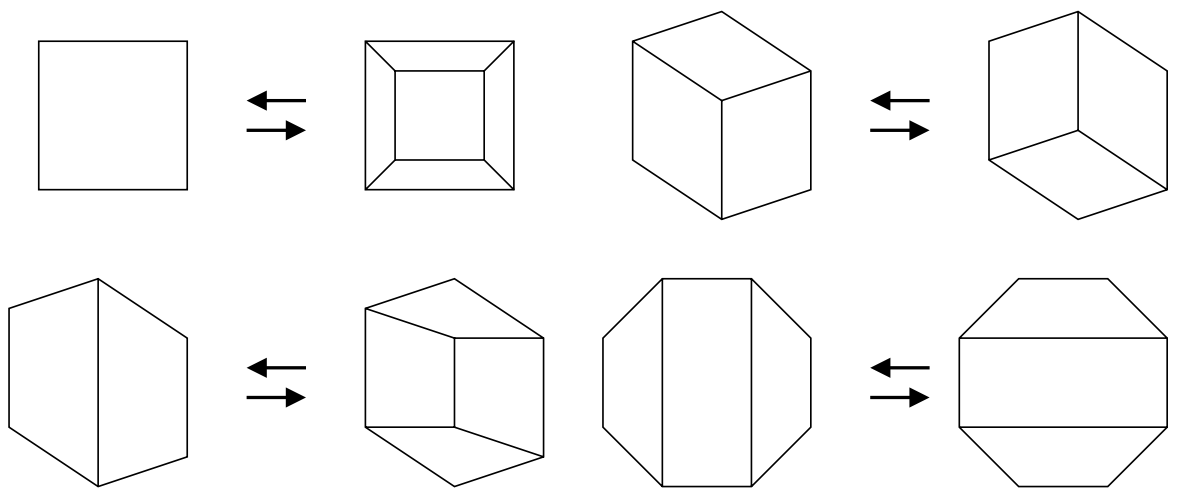

Fig. 4 Bubble moves for $k=2$

\section{Generalized Lovász Conjecture}

In this section we demonstrate how the groupoid of projectivities introduced by Joswig can be used as a basis for constructing parallel transport of graphs and more general Hom-complexes. In this framework we develop a general conceptual approach to the Lovász Hom-conjecture, recently resolved by E. Babson and D. Kozlov [4, 34], and extend their result both from graphs to simplicial complexes (Theorem 4.21, Corollary 4.23) and to other test graphs (Theorem 4.24).

\subsection{The Lovász Conjecture—an Overview}

One of central themes in topological combinatorics, after the landmark paper of Laszlo Lovász [36] where he proved the classical Kneser conjecture, has been the study and applications of graph complexes.

The underlying theme is to explore how the topological complexity of a graph complex $X(G)$ reflects in the combinatorial complexity of the graph $G$ itself. The results we are usually interested in come in the form of inequalities $\alpha(X(G)) \leq \xi(G)$, or equivalently in the form of implications

$$
\alpha(X(G)) \geq p \Rightarrow \xi(G) \geq q,
$$

where $\alpha(X(G))$ is a topological invariant of $X(G)$, while $\xi(G)$ is a combinatorial invariant of the graph $G$.

The most interesting candidate for the invariant $\xi$ has been the chromatic number $\chi(G)$ of $G$, while the role of the invariant $\alpha$ was played by the "connectedness" of $X(G)$, its equivariant index, the height of an associated characteristic cohomology class etc., see $[34,37,38,48]$ for recent accounts.

The famous result of Lovász quoted above is today usually presented in the form of an implication

$$
\operatorname{Hom}\left(K_{2}, G\right) \text { is } k \text {-connected } \Rightarrow \chi(G) \geq k+3,
$$

where $\operatorname{Hom}\left(K_{2}, G\right)$ is (together with the neighborhood complex, "box complex" etc.) one of the avatars of the homotopically unique $\mathbb{Z}_{2}$-graph complex of $G[38,48]$. This 
complex is a special case of a general graph complex $\operatorname{Hom}(H, G)$ (also introduced by L. Lovász), a cell complex which functorially depends on the input graphs $H$ and $G$.

An outstanding conjecture in this area, referred to as the "Lovász conjecture", was that one obtains a better bound if the graph $K_{2}$ in (1) is replaced by an odd cycle $C_{2 r+1}$. More precisely, Lovász conjectured that

$$
\operatorname{Hom}\left(C_{2 r+1}, G\right) \text { is } k \text {-connected } \Rightarrow \chi(G) \geq k+4 \text {. }
$$

This conjecture was confirmed by Babson and Kozlov in [4], see also [34] for a more detailed exposition and $[44,49]^{1}$ for subsequent developments.

Our objective is to develop methods which offer both a simplified approach to the proof of implication (2), at least in the case when $k$ is odd, and providing new insight, open a possibility of proving similar results for other classes of (hyper)graphs and simplicial complexes.

An example of such a result is Theorem 4.21. One of its corollaries is the implication

$$
\operatorname{Hom}(\Gamma, K) \text { is } k \text {-connected } \Rightarrow \chi(K) \geq k+d+3
$$

which, under a suitable assumption on the complex $\Gamma$ and the assumption that integer $k$ is odd, extends (2) to the case of pure $d$-dimensional simplicial complexes. A consequence of (3) is Theorem 4.24 which provides a "receipt" how to, for odd $k$, generate new examples of "homotopy test graphs" [34]. That is, graphs $T$ which satisfy the formula

$$
\operatorname{Hom}(T, G) \text { is } k \text {-connected } \Rightarrow \chi(G) \geq k+\chi(T)+1 \text {. }
$$

Examples include the lower three complexes depicted in Fig. 5 which all have chromatic number 4 and clique number 3 .

\subsection{Parallel Transport of Hom-Complexes}

For the definition and basic properties of graph complexes $\operatorname{Hom}(G, H)$ the reader is referred to [34]. More general Hom-complexes associated to simplicial complexes $K$ and $L$ are introduced in Sect. 4.4. Section 2.1 can be used as a glossary for basic concepts like "bundles", "parallel transport", "connection" etc.

\subsubsection{Natural Bundles and Groupoids Over Simplicial Complexes}

Suppose that $K$ and $L$ are finite simplicial complexes and let $k$ be an integer such that $0 \leq k \leq \operatorname{dim}(K)$. Denote by $V(K)=K^{(0)}$ the set of all vertices of $K$. Let $S_{k}=S_{k}(K)$ be the set of all $k$-dimensional simplices in $K$. Define a bundle $\mathcal{F}_{k}^{L}: S_{k} \rightarrow$ Top by the formula

$$
\mathcal{F}_{k}^{L}(\sigma)=\operatorname{Hom}(\sigma, L) \cong \operatorname{Hom}\left(\Delta^{[k+1]}, L\right),
$$

where $\operatorname{Hom}(\sigma, L)$ is one of the Hom-complexes introduced in Sect. 4.4.1 and $\Delta^{[k+1]}$ is the simplex with $[k+1]=\{1, \ldots, k+1\}$ as the set of vertices. By definition a

\footnotetext{
${ }^{1}$ This is a preliminary report which served as a basis for the current Sect. 4 .

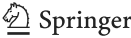


typical cell in $\operatorname{Hom}\left(\Delta^{[k+1]}, L\right)$ is of the form $e=\sigma_{1} \times \cdots \times \sigma_{k+1} \in L^{k+1}$ where $\left\{\sigma_{i}\right\}_{i=1}^{k+1}$ is a collection of nonempty simplices in $L$ such that $\sigma_{i} \cap \sigma_{j}=\emptyset$ for $i \neq j$ and

$$
V\left(\sigma_{1}\right) * V\left(\sigma_{2}\right) * \cdots * V\left(\sigma_{k+1}\right) \subset L .
$$

The corresponding cell in $\operatorname{Hom}(\sigma, L)$ is described by a function $\eta: V(\sigma) \rightarrow$ $2^{V(L)} \backslash\{\emptyset\}$ such that $\eta\left(v_{1}\right) \cap \eta\left(v_{2}\right)=\emptyset$ for $v_{1} \neq v_{2}$ and

$$
*_{v \in V(\sigma)} \eta(v)=\bigcup_{v \in V(\sigma)} \eta(v) \in L .
$$

Example The complex $\operatorname{Hom}\left(\sigma^{k}, \sigma^{m}\right) \cong \operatorname{Hom}\left(\Delta^{[k+1]}, \Delta^{[m+1]}\right)$ is well known in combinatorics as the $(k+1)$-fold "deleted product" $\left(\Delta^{[m+1]}\right)_{\delta}^{k+1}$ of $\Delta^{[m+1]}[37$, Sect. 6.3]. It is well known that for $k=1$ the associated deleted square $\left(\Delta^{[m+1]}\right)_{\delta}^{2}$ is homeomorphic to a $(m-1)$-dimensional sphere. In other words, $\mathcal{F}_{1}^{\sigma^{m}}: S_{1}(K) \rightarrow$ Top is a spherical bundle naturally associated to the simplicial complex $K$.

Our next goal, in the spirit of Sect. 2.1, is to identify a groupoid on the set $S_{k}$ which acts on the bundle $\mathcal{F}_{k}^{L}$, i.e. a groupoid which provides a parallel transport of fibres of the bundle $\mathcal{F}_{k}^{L}$. It turns out (Proposition 4.1) that this groupoid is precisely the $\mathcal{E}$-groupoid $\mathcal{E}\left(K^{(k)}\right)$, associated to the $k$-skeleton of $K$, introduced in Sect. 2.

It came as a pleasant surprise that this groupoid has already appeared in geometric combinatorics [28, 29]. Indeed, the groups of projectivities M. Joswig introduced and studied in these papers are just the holonomy groups of a groupoid which we call the $k$ th groupoid of projectivities of $K$ and denote by $\mathcal{J}_{k}(K)$. In these and subsequent papers [25-27], the groups of projectivities found interesting applications to toric manifolds, branched coverings over $S^{3}$, colorings of simple polytopes etc. The following excerpt from [28] reveals the role this construction played as a key motivating example for more general concepts introduced in Sect. 2.

“. .. For each ridge $\rho$ contained in two facets $\sigma, \tau$, there is a unique vertex $v(\sigma, \tau)$ which is contained in $\sigma$ but not in $\tau$. We define the perspectivity $\langle\sigma, \tau\rangle$ :

$\sigma \rightarrow \tau$ by setting

$$
w \mapsto \begin{cases}v(\tau, \sigma) & \text { if } w=v(\sigma, \tau), \\ w, & \text { otherwise. }\end{cases}
$$

... The projectivity $\langle g\rangle$ from $\sigma_{1}$ to $\sigma_{n}$ along $g$ is a concatenation

$$
\langle g\rangle=\left\langle\sigma_{0} . \sigma_{1}, \ldots, \sigma_{n}\right\rangle=\left\langle\sigma_{0}, \sigma_{1}\right\rangle\left\langle\sigma_{1}, \sigma_{2}\right\rangle \cdots\left\langle\sigma_{n-1}, \sigma_{n}\right\rangle
$$

of perspectivities. The map $\langle g\rangle$ is a bijection from $\sigma_{0}$ to $\sigma_{n} \ldots$,

Proposition 4.1 For each simplicial complex $K$ and an auxiliary "coefficient" complex $L$, there exists a canonical $\mathcal{J}_{k}(K)$-connection $\mathcal{P}^{L}=\mathcal{P}_{K, k}^{L}$ on the bundle $\mathcal{F}_{k}^{L}$. In other words the function $\mathcal{F}_{k}^{L}: S_{k} \rightarrow$ Top can be enriched (extended) to a functor

$$
\mathcal{F}_{k}^{L}: \mathcal{J}_{k}(K) \rightarrow \text { Top }
$$

where $\mathcal{J}_{k}(K) \cong \mathcal{E}_{k}(K)$ is the kth Joswig's groupoid of projectivities of $K$. 
Proof If $\overrightarrow{\sigma_{0} \sigma_{1}}$ is a perspectivity from $\sigma_{0}$ to $\sigma_{1}$ (a "flip" in the language of Sect. 2.2) and if $\eta: V\left(\sigma_{1}\right) \rightarrow 2^{V(L)} \backslash\{\emptyset\}$ is a cell in $\operatorname{Hom}(\sigma, L)$, then $\mathcal{P}^{L}: \mathcal{F}^{L}\left(\sigma_{1}\right) \rightarrow \mathcal{F}^{L}\left(\sigma_{0}\right)$ is the map defined by $\mathcal{P}^{L}\left(\overrightarrow{\sigma_{0} \sigma_{1}}\right)(\eta):=\overrightarrow{\sigma_{0} \sigma_{1}} * \eta$. More generally, if $\mathfrak{p}=\overrightarrow{\sigma_{0} \sigma_{1}} * \overrightarrow{\sigma_{1} \sigma_{2}} *$ $\cdots * \overrightarrow{\sigma_{n-1} \sigma_{n}}$ is a "projectivity" between $\sigma_{0}$ and $\sigma_{n}$, then

$$
\mathcal{P}^{L}(\mathfrak{p})=\mathcal{P}^{L}\left(\overrightarrow{\sigma_{0} \sigma_{1}}\right) * \mathcal{P}^{L}\left(\overrightarrow{\sigma_{1} \sigma_{2}}\right) * \cdots * \mathcal{P}^{L}\left(\overrightarrow{\sigma_{n-1}} \overrightarrow{\sigma_{n}}\right),
$$

or in other words

$$
\mathcal{P}^{L}(\mathfrak{p})(\eta)=\overrightarrow{\sigma_{0} \sigma_{1}} * \overrightarrow{\sigma_{1} \sigma_{2}} * \cdots * \overrightarrow{\sigma_{n-1} \sigma_{n}} * \eta=\mathfrak{p} * \eta
$$

which demonstrates in passing that the map $\mathcal{P}^{L}(\mathfrak{p})$ depends only on the morphism $\mathfrak{p}: \sigma_{0} \rightarrow \sigma_{n}$ alone, and not on the associated path $\sigma_{0}, \ldots, \sigma_{n}$.

\subsubsection{Parallel Transport of Graph Complexes}

The main motivation for introducing the parallel transport of Hom-complexes is the Lovász conjecture and its ramifications. This is the reason why the case of graphs and the graph complexes deserves a special attention. Additional justification for emphasizing graphs comes from the fact that graph complexes $\operatorname{Hom}(G, H)$ have been studied in numerous papers and today form a well-established part of graph theory and topological combinatorics. The situation with simplicial complexes is quite the opposite. In order to extend the theory of Hom-complexes from graphs to the category of simplicial complexes, many concepts should be generalized and the corresponding facts established in a more general setting. One is supposed to recognize the main driving forces and to isolate the most desirable features of the theory. A result should be a dictionary/glossary of associated concepts, cf. Table 1. Consequently, Sect. 4.2.2 should be viewed as an important preliminary step, leading to the more general theory developed in Sects. 4.4 and 4.5.

In order to simplify the exposition we assume, without a serious loss of generality, that all graphs $G=(V(G), E(G))$ are without loops and multiple edges. In short, graphs are one-dimensional simplicial complexes. Let $G_{\overline{x y}} \cong K_{2}$ be the restriction of $G$ on the edge $\overline{x y} \in E(G)$.

Following the definitions from Sect. 4.2.1 the map

$$
\mathcal{F}^{H}: E(G) \longrightarrow T o p,
$$

Table 1 Graphs vs. simplicial complexes

Dictionary

\begin{tabular}{ll} 
Graphs & Simplicial complexes \\
Trees & Tree-like complexes \\
Foldings of graphs & Vertex collapsing of complexes \\
Graph homomorphisms & Nondegenerate simplicial maps \\
Hom $(G, H)$ & Hom $(K, L)$ \\
Chromatic number $\chi(G)$ & Chromatic number $\chi(K)$ \\
\hline
\end{tabular}


where $\mathcal{F}^{H}(\overline{x y})=\mathcal{F}_{\overline{x y}}^{H}:=\operatorname{Hom}\left(G_{\overline{x y}}, H\right)$, can be thought of as a "bundle" over the graph $G$, with $\mathcal{F}_{\overline{x y}}^{H}$ in the role of the "fibre" over the edge $\overline{x y}$. More generally, given a class $\mathcal{C}$ of subgraphs of $G$, say the subtrees, the chains, the $k$-cliques etc., one can define an associated "bundle" $\mathcal{F}_{\mathcal{C}}^{H}: \mathcal{C} \rightarrow$ Top by a similar formula $\mathcal{F}_{\mathcal{C}}^{H}(\Gamma):=$ $\operatorname{Hom}(\Gamma, H)$, where $\Gamma \in \mathcal{C}$.

The parallel transport $\mathcal{P}^{H}$, for a given graph (one-dimensional, simplicial complex) $H$, is a specialization of the parallel transport $\mathcal{P}^{L}$ introduced in Sect. 4.2.1. For example, if $\overrightarrow{e_{1} e_{2}}$ is the flip (perspectivity) between adjacent edges $e_{1}=\overline{x_{0} x_{1}}$ and $e_{2}=\overline{x_{1} x_{2}}$ in $G$, and if $\eta:\left\{x_{1}, x_{2}\right\} \rightarrow 2^{V(H)} \backslash\{\emptyset\}$ is a cell in $\mathcal{F}_{\overline{x_{1} x_{2}}}^{H}=\operatorname{Hom}\left(G_{\overline{x_{1} x_{2}}}, H\right)$, then $\eta^{\prime}:=\mathcal{P}^{H}\left(\overrightarrow{e_{1} e_{2}}\right)(\eta):\left\{x_{0}, x_{1}\right\} \rightarrow 2^{V(H)} \backslash\{\emptyset\}$ is defined by

$$
\eta^{\prime}\left(x_{0}\right):=\eta\left(x_{2}\right) \quad \text { and } \quad \eta^{\prime}\left(x_{1}\right):=\eta\left(x_{1}\right) .
$$

Fundamental observation The constructions of the connections $\mathcal{P}^{L}$, respectively $\mathcal{P}^{H}$, are quite natural and elementary, but it is Proposition 4.3, and its more general relative Proposition 4.16, that serve as an actual justification for the introduction of these objects. Proposition 4.3 allows us to analyze the parallel transport of homotopy types of maps from the complex $\operatorname{Hom}(G, H)$ to complexes $\operatorname{Hom}\left(G_{e}, H\right)$, where $e \in E(G)$, providing a key for a resolution of the Lovász conjecture in the case when $k$ is an odd integer.

Implicit in the proof of Proposition 4.3 is the theory of folds of graphs and the analysis of natural morphisms between graph complexes $\operatorname{Hom}(T, H)$, where $T$ is a tree, as developed in [3, 32, 33]. This theory is one of essential ingredients in the Babson and Kozlov spectral sequence approach to the solution of Lovász conjecture. Some of these results are summarized in Proposition 4.2, in a form suitable for application to Proposition 4.3.

As usual $L_{m}$ is the graph-chain of vertex-length $m$, while $L_{x_{1}, \ldots, x_{m}}$ is the graph isomorphic to $L_{m}$ defined on a linearly ordered set of vertices $x_{1}, \ldots, x_{m}$. In this context the "chain-flip" is a generic name for the automorphism $\sigma: L_{x_{1}, \ldots, x_{m}} \rightarrow L_{x_{1}, \ldots, x_{m}}$ of the graph-chain such that $\sigma\left(x_{j}\right)=x_{m-j+1}$ for each $j$.

Proposition 4.2 Suppose that $e_{1}=\overline{x_{0} x_{1}}$ and $e_{2}=\overline{x_{1} x_{2}}$ are two distinct, adjacent edges in the graph G. Let $\sigma: L_{x_{0} x_{1} x_{2}} \rightarrow L_{x_{0} x_{1} x_{2}}$ be the chain-flip automorphism of $L_{x_{0} x_{1} x_{2}}$ and $\widehat{\sigma}$ the associated auto-homeomorphism of Hom $\left(L_{x_{0} x_{1} x_{2}}, H\right)$. Suppose that $\gamma_{i j}: L_{x_{i} x_{j}} \rightarrow L_{x_{0} x_{1} x_{2}}$ is an obvious embedding and $\widehat{\gamma}_{i j}$ the associated map of graph complexes. Then,

(a) The induced map $\widehat{\sigma}: \operatorname{Hom}\left(L_{x_{0} x_{1} x_{2}}, H\right) \rightarrow \operatorname{Hom}\left(L_{x_{0} x_{1} x_{2}}, H\right)$ is homotopic to the identity map I, and

(b) The diagram

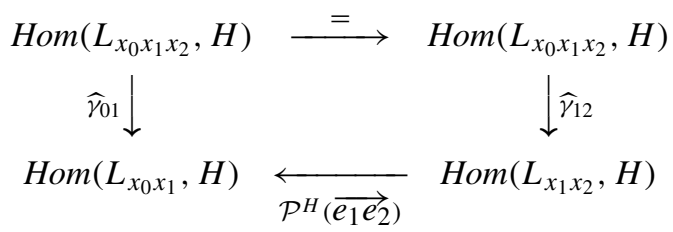

is commutative up to homotopy 
Proof Both statements are corollaries of Babson and Kozlov analysis of complexes $\operatorname{Hom}(T, H)$, where $T$ is a tree, and morphisms $\widehat{e}: \operatorname{Hom}(T, H) \rightarrow \operatorname{Hom}\left(T^{\prime}, H\right)$, where $T^{\prime}$ is a subtree of $T$ and $e: T^{\prime} \rightarrow T$ the associated embedding.

Our starting point is an observation that both $L_{x_{0} x_{1}}$ and $L_{x_{1} x_{2}}$ are retracts of the graph $L_{x_{0} x_{1} x_{2}}$ in the category of graphs and graph homomorphisms. The retraction homomorphisms $\phi_{i j}: L_{x_{0} x_{1} x_{2}} \rightarrow L_{x_{i} x_{j}}$, where $\phi_{01}\left(x_{0}\right)=x_{0}, \phi_{01}\left(x_{1}\right)=x_{1}$, $\phi_{01}\left(x_{2}\right)=x_{0}$ and $\phi_{12}\left(x_{0}\right)=x_{2}, \phi_{12}\left(x_{1}\right)=x_{1}, \phi_{12}\left(x_{2}\right)=x_{2}$ are examples of foldings of graphs. By the general theory $[3,33]$, the maps $\widehat{\gamma}_{i j}: \operatorname{Hom}\left(L_{x_{0} x_{1} x_{2}}, H\right) \rightarrow$ $\operatorname{Hom}\left(L_{x_{i} x_{j}}, H\right)$ and $\widehat{\phi}_{i j}: \operatorname{Hom}\left(L_{x_{i} x_{j}}, H\right) \rightarrow \operatorname{Hom}\left(L_{x_{0} x_{1} x_{2}}, H\right)$ are homotopy equivalences. Actually $\widehat{\gamma}_{i j}$ is a deformation retraction and $\widehat{\phi}_{i j}$ is the associated embedding such that $\widehat{\gamma}_{i j} \circ \widehat{\phi}_{i j}=I$ is the identity map.

Part (a) of the proposition is an immediate consequence of the fact that $\phi_{01} \circ \sigma \circ$ $\gamma_{01}: L_{x_{0} x_{1}} \rightarrow L_{x_{0} x_{1}}$ is an identity map. It follows that $\widehat{\gamma}_{01} \circ \widehat{\sigma} \circ \widehat{\phi}_{01}=I$, and in light of the fact that $\widehat{\gamma}_{01}$ and $\widehat{\phi}_{01}$ are homotopy inverses to each other, we conclude that $\widehat{\sigma} \simeq I$.

For part (b) we begin with an observation that $\phi_{12} \circ \sigma \circ \gamma_{01}=\overrightarrow{e_{1} e_{2}}$. Then, $\mathcal{P}^{H}\left(\overrightarrow{e_{1} e_{2}}\right)=\widehat{\gamma}_{01} \circ \widehat{\sigma} \circ \widehat{\phi}_{12}$, and as a consequence of $\widehat{\sigma} \simeq I$ and the fact that $\widehat{\phi}_{12} \circ \widehat{\gamma}_{12} \simeq I$, we conclude that

$$
\mathcal{P}^{H}\left(\overrightarrow{e_{1} e_{2}}\right) \circ \widehat{\gamma}_{12}=\widehat{\gamma}_{01} \circ \widehat{\sigma} \circ \widehat{\phi}_{12} \circ \widehat{\gamma}_{12} \simeq \widehat{\gamma}_{01}
$$

Proposition 4.3 Suppose that $x_{0}, x_{1}, x_{2}$ are distinct vertices in $G$ such that $\overline{x_{0} x_{1}}$, $\overline{x_{1} x_{2}} \in E(G)$. Let $\alpha_{i j}: G_{x_{i} x_{j}} \rightarrow G$ be the inclusion map of graphs and $\widehat{\alpha}_{i j}$ the associated map of $\operatorname{Hom}(\cdot, H)$ complexes. Then the following diagram commutes up to a homotopy

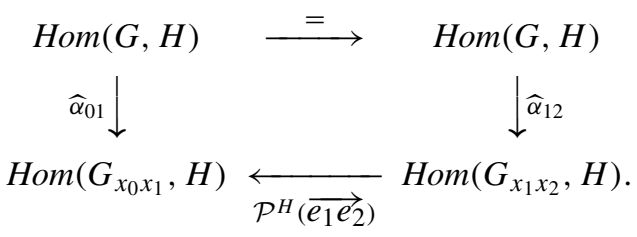

Proof The diagram (8) can be factored as

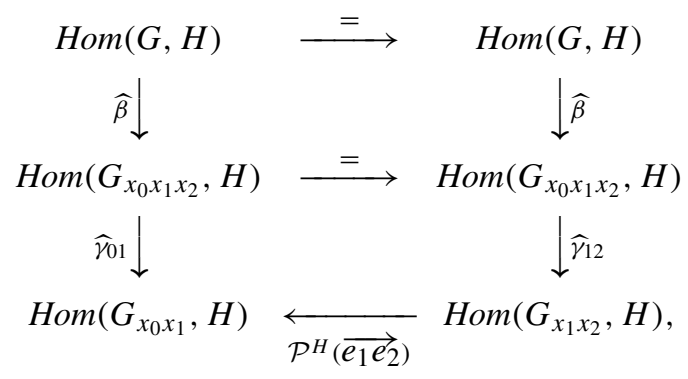

where $\beta$ and $\gamma_{i j}$ are obvious inclusions of indicated graphs such that $\alpha_{i j}=\beta \circ \gamma_{i j}$. Then the result is a direct consequence of Proposition 4.2(b). 


\subsection{Babson-Kozlov-Lovász Result for Odd $k$}

The proof [4] of Lovász conjecture splits into two main branches, corresponding to the parity of a parameter $n$, where $n$ is an integer which enters the stage as the size of the vertex set of the complete graph $K_{n}$.

The first branch relies on Theorem 2.3 (loc. cit.), more precisely on part (b) of this result, while the second branch is founded on Theorem 2.6. Both theorems are about the topology of the graph complex $\operatorname{Hom}\left(C_{2 r+1}, K_{n}\right)$. Theorem 2.3(b) is a statement about the height of the first Stiefel-Whitney class, ${ }^{2}$ equivalently the Conner-Floyd index [14] of the $\mathbb{Z}_{2}$-space $\operatorname{Hom}\left(C_{2 r+1}, K_{n}\right)$. Theorem 2.6 claims that for $n$ even, $2 \iota_{K_{n}}^{*}$ is a zero homomorphism where

$$
\iota_{K_{n}}^{*}: \widetilde{H}^{*}\left(\operatorname{Hom}\left(K_{2}, K_{n}\right) ; \mathbb{Z}\right) \longrightarrow \widetilde{H}^{*}\left(\operatorname{Hom}\left(C_{2 r+1}, K_{n}\right) ; \mathbb{Z}\right)
$$

is the homomorphism associated to the continuous map

$$
\iota_{K_{n}}: \operatorname{Hom}\left(C_{2 r+1}, K_{n}\right) \rightarrow \operatorname{Hom}\left(K_{2}, K_{n}\right),
$$

which in turn comes from the inclusion $K_{2} \hookrightarrow C_{2 r+1}$.

The central idea of our approach is an observation that Theorem 2.6 can be incorporated into a more general scheme, involving the "parallel transport" of graph complexes over graphs.

Theorem 4.4 Suppose that $\alpha: K_{2} \rightarrow C_{2 r+1}$ is an inclusion map, $\beta: K_{2} \rightarrow K_{2} a$ nontrivial automorphism of $K_{2}$, and

$$
\widehat{\alpha}: \operatorname{Hom}\left(C_{2 r+1}, H\right) \rightarrow \operatorname{Hom}\left(K_{2}, H\right), \quad \widehat{\beta}: \operatorname{Hom}\left(K_{2}, H\right) \rightarrow \operatorname{Hom}\left(K_{2}, H\right)
$$

the associated maps of graph complexes. Then the following diagram is commutative up to a homotopy

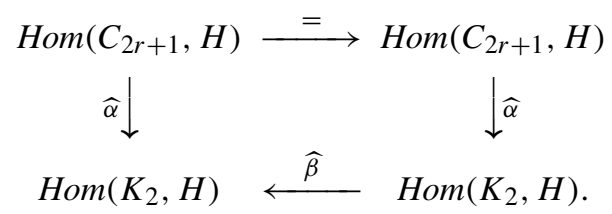

Proof Assume that the consecutive vertices of $G=C_{2 r+1}$ are $x_{0}, x_{1}, \ldots, x_{2 r}$ and let $e_{i}=\overline{x_{i-1} x_{i}}$ be the associated sequence of edges where by convention $e_{2 r+1}=\overline{x_{2 r} x_{0}}$. Identify the graph $K_{2}$ to the subgraph $G_{x_{0} x_{1}}$ of $G=C_{2 r+1}$.

By iterating Proposition 4.3 we observe that the diagram

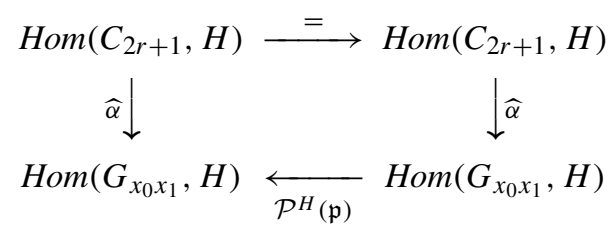

\footnotetext{
${ }^{2}$ Subsequently C. Schultz discovered [44] a powerful new way of evaluating this invariant, leading to a much shorter proof of Lovász conjecture.
} 
is commutative up to a homotopy, where $\mathfrak{p}=\overrightarrow{e_{1} e_{2}} * \cdots * \overrightarrow{e_{2 r+1}} \vec{e}_{1}$. The proof is completed by the observation that $\mathfrak{p}=\beta$ in the groupoid $\mathcal{J}\left(C_{2 r+1}\right) \cong \mathcal{E}\left(C_{2 r+1}\right)$.

Theorem 2.6 from [4], the key for the proof of Lovász conjecture for odd $k$, is an immediate consequence of Theorem 4.4 .

Corollary 4.5 ([4], Theorem 2.6) If $n$ is even, then $2 \cdot \iota_{K_{n}}^{*}$ is a 0 -map where $\iota_{K_{n}}^{*}$ is the map described in line (10).

Proof It is sufficient to observe that for $H=K_{n}, \operatorname{Hom}\left(K_{2}, K_{n}\right) \cong S^{n-2}$ is an evendimensional sphere and that the automorphism $\widehat{\beta}$ from the diagram (11) is in this case essentially an antipodal map. It follows that $\widehat{\beta}$ changes the orientation of $\operatorname{Hom}\left(K_{2}, K_{n}\right)$ and as a consequence $\iota_{K_{n}}^{*}=-\iota_{K_{n}}^{*}$.

\subsection{Generalizations and Ramifications}

In this section we extend the results from Sect. 4.2.2 to the case of simplicial complexes. This generalization is based on the following basic principles.

Graphs are viewed as one-dimensional simplicial complexes. Graph homomorphisms are special cases of nondegenerate simplicial maps of simplicial complexes, see $[27,28]$ and Definition 2.4. The definition of $\operatorname{Hom}(G, H)$ is extended to the case of $H o m$-complexes $\operatorname{Hom}(K, L)$ of simplicial complexes $K$ and $L$. The groupoids needed for the definition of the parallel transport of Hom-complexes are described in Sect. 4.2.1. Theory of folds for graph complexes [3, 33] is extended in Sect. 4.4.4 to the case of Hom-complexes in sufficient generality to allow "parallel transport" of homotopy types of maps between graph complexes. This development eventually leads to Theorem 4.21 which extends Theorem 4.4 to the case of Hom-complexes $\operatorname{Hom}(K, L)$ and represents the currently final stage in the evolution of Theorem 2.6 from [4].

\subsubsection{From $\operatorname{Hom}(G, H)$ to $\operatorname{Hom}(K, L)$}

Suppose that $K \subset 2^{V(K)}$ and $L \subset 2^{V(L)}$ are two (finite) simplicial complexes, on the sets of vertices $V(K)$ and $V(L)$, respectively.

Definition 4.6 The set of all nondegenerate simplicial maps from $K$ to $L$ is denoted by $\operatorname{Hom}_{0}(K, L)$ where a map $f: K \rightarrow L$ is nondegenerate (Definition 2.4) if it is injective on simplices.

Definition 4.7 $\operatorname{Hom}(K, L)$ is a cell complex with the cells indexed by the functions $\eta: V(K) \rightarrow 2^{V(L)} \backslash\{\emptyset\}$ such that

(1) For each two vertices $u \neq v$, if $\{u, v\} \in K$, then $\eta(u) \cap \eta(v)=\emptyset$.

(2) For each simplex $\sigma \in K$, the join $*_{v \in V(\sigma)} \eta(v) \subset \Delta^{V(L)}$ of all sets (or 0 -dimensional complexes) $\eta(v)$ is a subcomplex of $L$.

More precisely, each function $\eta$ satisfying conditions (1) and (2) defines a cell $c_{\eta}:=\prod_{v \in V(K)} \Delta^{\eta(v)}$ in $\operatorname{Hom}(K, L) \subset \prod_{v \in V(K)} \Delta^{V(L)}$ where by definition $\Delta^{S}$ is an (abstract) simplex spanned by vertices in $S$. 
The following example shows that $\operatorname{Hom}(K, L)$ are close companions of graph complexes $\operatorname{Hom}(G, H)$.

Example 4.8 The definition of the complex $\operatorname{Hom}(K, L)$ is a natural extension of $\operatorname{Hom}(G, H)$ and reduces to it if $K$ and $L$ are one-dimensional complexes. Moreover,

$$
\operatorname{Hom}(G, H) \cong \operatorname{Hom}(\operatorname{Clique}(G), \text { Clique }(H)),
$$

where Clique $(\Gamma)$ is the simplicial complex of all cliques in a graph $\Gamma$.

Remark 4.9 The set $\operatorname{Hom}_{0}(K, L)$ is easily identified as the 0-dimensional skeleton of the cell-complex $\operatorname{Hom}(K, L)$. Moreover, the reader familiar with [34] can easily check that $\operatorname{Hom}(K, L)$ is determined by the family $M=\operatorname{Hom}_{0}(K, L)$ in the sense of Definition 2.2.1 from that paper.

\subsubsection{Functoriality of $\operatorname{Hom}(K, L)$}

The construction of $\operatorname{Hom}(K, L)$ is functorial in the sense that if $f: K \rightarrow K^{\prime}$ is a nondegenerate simplicial map of complexes $K$ and $K^{\prime}$, then there is an associated continuous map $\widehat{f}: \operatorname{Hom}\left(K^{\prime}, L\right) \rightarrow \operatorname{Hom}(K, L)$ of Hom-complexes. Indeed, if $\eta$ : $V\left(K^{\prime}\right) \rightarrow 2^{V(L)} \backslash\{\emptyset\}$ is a multivalued function indexing a cell in $\operatorname{Hom}\left(K^{\prime}, L\right)$, then it is not difficult to check that $\eta \circ f: V(K) \rightarrow 2^{V(L)} \backslash\{\emptyset\}$ is a cell in $\operatorname{Hom}(K, L)$.

Even more important is the functoriality of $\operatorname{Hom}(K, L)$ with respect to the second variable since this implies the functoriality of the bundle $\mathcal{F}_{k}^{L}$.

Proposition 4.10 Suppose that $g: L \rightarrow L^{\prime}$ is a nondegenerate, simplicial map of simplicial complexes $L$ and $L^{\prime}$. Then there exists an associated map

$$
\widehat{g}: \operatorname{Hom}(K, L) \rightarrow \operatorname{Hom}\left(K, L^{\prime}\right)
$$

Proof Assume that $\eta: V(K) \rightarrow 2^{V(L)} \backslash\{\emptyset\}$ is a cell in $\operatorname{Hom}(K, L)$. Then $g \circ \eta$ : $V(K) \rightarrow 2^{V\left(L^{\prime}\right) \backslash\{\emptyset\}}$ is a cell in $\operatorname{Hom}\left(K, L^{\prime}\right)$. Suppose $u$ and $v$ are distinct vertices in $V(K)$. By assumption $\eta(u) \cap \eta(v)=\emptyset$. We deduce from here that $g(\eta(u)) \cap$ $g(\eta(v)) \neq \emptyset$, otherwise $g$ would be a degenerated simplicial map. The second condition from Definition 4.7 is checked by a similar argument.

\subsubsection{Chromatic Number $\chi(K)$ and Its Relatives}

The chromatic number $\chi(K)$ of a simplicial complex $K$ is

$$
\inf \left\{m \in \mathbb{N} \mid \operatorname{Hom}_{0}\left(K, \Delta^{[m]}\right) \neq \emptyset\right\} .
$$

In other words $\chi(K)$ is the minimum number $m$ such that there exists a nondegenerate simplicial map $f: K \rightarrow \Delta^{[m]}$. It is not difficult to check that $\chi(K)=\chi\left(G_{K}\right)$, where $G_{K}=\left(K^{(0)}, K^{(1)}\right)$ is the vertex-edge graph of the complex $K$. In particular $\chi(K)$ reduces to the usual chromatic number if $K$ is a graph, that is if $K$ is a onedimensional simplicial complex. 
Aside from the usual chromatic number $\chi(G)$, there are many related colorful graph invariants $[23,34]$. Among the best known are the fractional chromatic number $\chi_{f}(G)$ and the circular chromatic number $\chi_{c}(G)$ of $G$. These and other related invariants are conveniently defined in terms of graph homomorphisms into graphs chosen from a suitable family $\mathcal{F}=\left\{G_{i}\right\}_{i \in I}$ of test graphs. Motivated by this we offer an extension of the chromatic number $\chi(K)$ in hope that some genuine, new invariants of simplicial complexes may arise this way.

Definition 4.11 Suppose that $\mathcal{F}=\left\{T_{i} \mid i \in I\right\}$ is a family of "test" simplicial complexes and let $\phi: I \rightarrow \mathbb{R}$ is a real-valued function. A $T_{i}$-coloring of $K$ is just a nondegenerate simplicial map $f: K \rightarrow T_{i}$ and $\chi_{(\mathcal{F}, \phi)}(K)$, the $(\mathcal{F}, \phi)$-chromatic number of $K$, is defined as the infimum of all weights $\phi(i)$ over all $T_{i}$-colorings,

$$
\chi_{(\mathcal{F}, \phi)}(K):=\inf \left\{\phi(i) \mid \operatorname{Hom}_{0}\left(K, T_{i}\right) \neq \emptyset\right\} .
$$

\subsubsection{Tree-Like Simplicial Complexes}

In the theory of $\operatorname{Hom}(K, L)$-complexes, the tree-like or vertex collapsible complexes are intended to play a role similar to that of trees in the theory of graph complexes $\operatorname{Hom}(G, H)$.

A pure, $d$-dimensional simplicial complex $K$ is shellable $[9,47]$, if there is a linear order $F_{1}, F_{2}, \ldots, F_{m}$ on the set of its facets, such that for each $j \geq 2$, the complex $F_{j} \cap\left(\bigcup_{i<j} F_{i}\right)$ is a pure $(d-1)$-dimensional subcomplex of the simplex $F_{j}$. The restriction $R_{j}$ of the facet $F_{j}$ is the minimal new face added to the complex $\bigcup_{k<j} F_{k}$ by the addition of the facet $F_{j}$. Let $r_{j}:=\operatorname{dim}\left(R_{j}\right) \in\{0,1, \ldots, d\}$ be the type of the facet $F_{j}$. If $r_{j} \neq d$ for each $j$, then the complex $K$ is collapsible. The collapsing process is just the shelling order read in the opposite direction. From this point of view, $R_{j}$ can be described as a free face in the complex $\bigcup_{i \leq j} F_{i}$, and the process of removing all faces $F$ such that $R_{j} \subset F \subset F_{j}$ is called an elementary $r_{j}$-collapse.

Definition 4.12 A pure $d$-dimensional simplicial complex $K$ is called tree-like or vertex collapsible if it is collapsible to a $d$-simplex with the use of elementary 0 -collapses alone. In other words, $K$ is shellable and for each $j \geq 2$ the intersection $F_{j} \cap\left(\bigcup_{i<j} F_{i}\right)$ is a proper face of $F_{j}$.

In order to establish analogues of Propositions 4.2 and 4.3 for $\operatorname{Hom}(K, L)$ complexes, we prove a result which shows that elementary vertex collapsing provides a good substitute and a partial generalization for the concept of "foldings" of graphs used in $[3,33]$ in the theory of graph complexes $\operatorname{Hom}(G, H)$.

Proposition 4.13 Suppose that the simplicial complex $K^{\prime}$ is obtained from $K$ by an elementary vertex collapse. In other words we assume that $K=\sigma \cup K^{\prime}, \sigma \cap K^{\prime}=\sigma^{\prime}$, where $\sigma$ is a simplex in $K$ and $\sigma^{\prime}$ a facet of $\sigma$. Assume that $\sigma^{\prime}$ is not maximal in $K^{\prime}$, i.e. that for some simplex $\sigma^{\prime \prime} \in K^{\prime}$ and a vertex $u \in \sigma^{\prime \prime}, \sigma^{\prime}=\sigma^{\prime \prime} \backslash\{u\}$. Then for any simplicial complex $L$, the inclusion map $\gamma: K^{\prime} \rightarrow K$ induces a homotopy equivalence

$$
\widehat{\gamma}: \operatorname{Hom}(K, L) \rightarrow \operatorname{Hom}\left(K^{\prime}, L\right)
$$


Proof Let $\{v\}=\sigma \backslash \sigma^{\prime}$. Aside from the inclusion map $\gamma: K^{\prime} \rightarrow K$, there is a retraction (folding) map $\rho: K \rightarrow K^{\prime}$, where $\rho(v)=u$ and $\left.\rho\right|_{K^{\prime}}=I_{K^{\prime}}$. Since $\rho \circ \gamma=I_{K^{\prime}}$, we observe that $\widehat{\gamma} \circ \widehat{\rho}=I d_{K^{\prime}}$ is the identity map on $\operatorname{Hom}\left(K^{\prime}, L\right)$, i.e. the complex $\operatorname{Hom}\left(K^{\prime}, L\right)$ is a retract of the complex $\operatorname{Hom}(K, L)$. It remains to be shown that $\widehat{\rho} \circ \widehat{\gamma} \simeq I d_{K}$ is homotopic to the identity map on $\operatorname{Hom}(K, L)$.

Note that if $\eta \in \operatorname{Hom}(K, L)$, then $\eta^{\prime}:=\widehat{\rho} \circ \widehat{\gamma}(\eta)$ is the function defined by

$$
\eta^{\prime}(w)= \begin{cases}\eta(w), & \text { if } w \neq v, \\ \eta(u), & \text { if } w=v .\end{cases}
$$

Let $\omega: \operatorname{Hom}(K, L) \rightarrow \operatorname{Hom}(K, L)$ be the map defined by

$$
\omega(\eta)(w)= \begin{cases}\eta(w), & \text { if } w \neq v, \\ \eta(u) \cup \eta(v), & \text { if } w=v .\end{cases}
$$

Note that $\omega$ is well defined since if a vertex $x$ is adjacent to $v$ it is also adjacent to $u$, hence the condition $\omega(\eta)(v) \cap \eta(x)=\emptyset$ is a consequence of $\eta(u) \cap \eta(x)=\emptyset=$ $\eta(v) \cap \eta(x)$.

Since for each $\eta \in \operatorname{Hom}(K, L)$ and each vertex $x \in K$,

$$
\eta(x) \subset \omega(\eta)(x) \supset \widehat{\rho} \circ \widehat{\gamma}(\eta)(x),
$$

by the Order Homotopy Theorem [8, 41, 42] all three maps $I d_{K}, \omega$ and $\widehat{\rho} \circ \widehat{\gamma}$ are homotopic. This completes the proof of the proposition.

Corollary 4.14 If $T$ is a d-dimensional, tree-like simplicial complex, then $\operatorname{Hom}(T, L)$ has the same homotopy type as the complex $\operatorname{Hom}\left(\Delta^{d}, L\right)$.

\subsubsection{Parallel Transport of Homotopy Types of Maps}

As in the case of graph complexes, the real justification for the introduction of the parallel transport of Hom-complexes comes from the fact that it preserves the homotopy type of the maps $\operatorname{Hom}(K, L) \rightarrow \operatorname{Hom}(\sigma, L)$. As a preliminary step we prove an analogue of Proposition 4.2.

Proposition 4.15 Suppose that $\sigma_{1}$ and $\sigma_{2}$ are two distinct, adjacent $k$-dimensional simplices in a finite simplicial complex $K$ which share a common $(k-1)$-dimensional simplex $\tau$. Let $\Sigma=\sigma_{1} \cup \sigma_{2}$. Let $\alpha: \Sigma \rightarrow \Sigma$ be the automorphism of $\Sigma$ which interchanges simplices $\sigma_{1}$ and $\sigma_{2}$ keeping the common face $\tau$ point-wise fixed.

Suppose that $\gamma_{i}: \sigma_{i} \rightarrow \Sigma$ is an obvious embedding and $\widehat{\gamma}_{i}$ the associated maps of Hom-complexes. Then,

(a) The induced map $\widehat{\alpha}: \operatorname{Hom}(\Sigma, L) \rightarrow \operatorname{Hom}(\Sigma, L)$ is homotopic to the identity map $I_{\Sigma}$, and

(b) The diagram

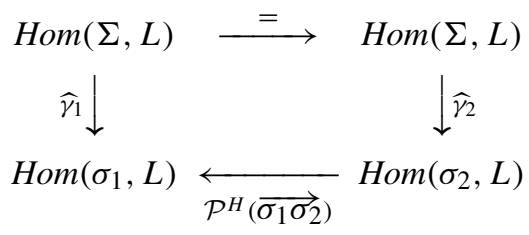

is commutative up to homotopy 
Proof By Proposition 4.13, both maps $\widehat{\gamma}_{i}: \operatorname{Hom}(\Sigma, L) \rightarrow \operatorname{Hom}\left(\sigma_{i}, L\right)$ for $i=1,2$ are homotopy equivalences. Let $\rho_{1}: \Sigma \rightarrow \sigma_{1}$ and $\rho_{2}: \Sigma \rightarrow \sigma_{2}$ be the folding maps. Then $\rho_{i} \circ \gamma_{i}=I_{\sigma_{i}}, \widehat{\gamma_{i}} \circ \widehat{\rho_{i}}=I$ and we conclude that $\widehat{\rho}_{i}: \operatorname{Hom}\left(\sigma_{i}, L\right) \rightarrow \operatorname{Hom}(\Sigma, L)$ is also a homotopy equivalence.

Part (a) of the proposition follows from the fact that $\rho_{1} \circ \alpha \circ \gamma_{1}=I_{\sigma_{1}}$ is an identity map. Indeed, an immediate consequence is that $\widehat{\gamma_{1}} \circ \widehat{\alpha} \circ \widehat{\rho_{1}}=I: \operatorname{Hom}\left(\sigma_{1}, L\right) \rightarrow$ $\operatorname{Hom}\left(\sigma_{1}, L\right)$ is also an identity map and, in light of the fact that $\widehat{\gamma_{1}}$ and $\widehat{\rho_{1}}$ are homotopy inverses to each other, we deduce that $\widehat{\alpha} \simeq I$.

For part (b) we begin with an observation that $\rho_{2} \circ \alpha \circ \gamma_{1}=\overrightarrow{\sigma_{1} \sigma_{2}}$. Then, $\mathcal{P}^{H}\left(\overrightarrow{\sigma_{1} \sigma_{2}}\right)=\widehat{\gamma_{1}} \circ \widehat{\alpha} \circ \widehat{\rho}_{2}$, and as a consequence of $\widehat{\alpha} \simeq I$ and the fact that $\widehat{\rho_{2}} \circ \widehat{\gamma}_{2} \simeq I$, we conclude that

$$
\mathcal{P}^{H}\left(\overrightarrow{\sigma_{1} \sigma_{2}}\right) \circ \widehat{\gamma_{2}}=\widehat{\gamma_{1}} \circ \widehat{\alpha} \circ \widehat{\rho}_{2} \circ \widehat{\gamma_{2}} \simeq \widehat{\gamma_{1}}
$$

Proposition 4.16 Suppose that $K$ and $L$ are finite simplicial complexes and $\sigma_{1}, \sigma_{2}$ a pair of adjacent (distinct), $k$-dimensional simplices in $K$. Let $\alpha_{i}: \sigma_{i} \rightarrow K$ be the embedding of $\sigma_{i}$ in $K$ and $\widehat{\alpha}_{i}: \operatorname{Hom}(K, L) \rightarrow \operatorname{Hom}\left(\sigma_{i}, L\right)$ the associated map of Hom-complexes. Then the following diagram commutes up to a homotopy.

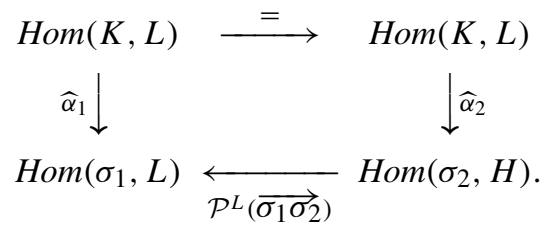

Proof Let $\Sigma:=\sigma_{1} \cup \sigma_{2}, \tau:=\sigma_{1} \cap \sigma_{2}$. Then $\alpha_{i}=\beta \circ \gamma_{i}$ where $\beta: \Sigma \rightarrow K$ and $\gamma_{i}: \sigma_{i} \rightarrow \Sigma$ are natural embeddings of complexes.

The diagram (13) can be factored as

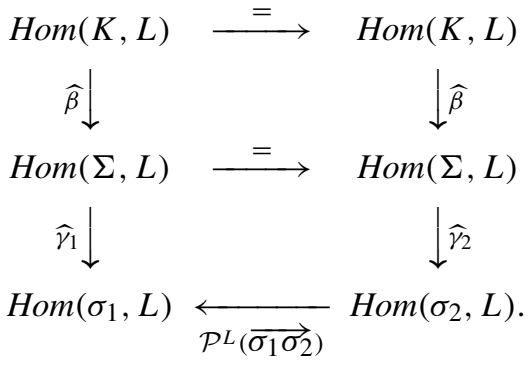

Then the result is a direct consequence of Proposition 4.15(b).

Corollary 4.17 Suppose that $K$ and $L$ are finite simplicial complexes, $\sigma a$ $k$-dimensional simplex in $K$ and $\alpha: \sigma \rightarrow K$ the associated embedding. Let $\tau \in$ $\Pi(K, \sigma)$. Then the following diagram commutes up to a homotopy.

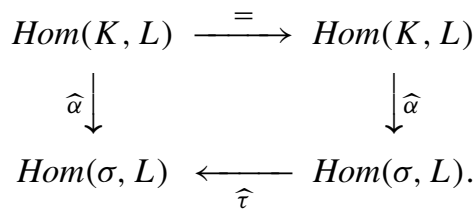




\subsection{A Generalized B-K-L Theorem}

In this section we prove the promised extension of the Babson-Kozlov-Lovász theorem. The graphs are replaced by pure $d$-dimensional simplicial complexes, while the role of the odd cycle $C_{2 r+1}$ is played by a complex $\Gamma$ which has some special symmetry properties in the sense of the following definition.

As usual, an involution $\omega: X \rightarrow X$ is the same as a $\mathbb{Z}_{2}$-action on $X$. An involution on a simplicial complex $\Gamma$ induces an involution on the complex $\operatorname{Hom}(\Gamma, L)$ for each simplicial complex $L$. For all other standard facts and definitions related to $\mathbb{Z}_{2}$-complexes the reader is referred to [37].

Definition 4.18 A pure $d$-dimensional simplicial complex $\Gamma$ is a $\Phi_{d}$-complex if it is a $\mathbb{Z}_{2}$-complex with an invariant $d$-simplex $\sigma=\left\{v_{0}, v_{1}, \ldots, v_{d}\right\}$ such that the restriction $\tau:=\left.\omega\right|_{\sigma}$ of the involution $\omega: \Gamma \rightarrow \Gamma$ on $\sigma$ is a nontrivial element of the group $\Pi(\Gamma, \sigma)$.

Remark 4.19 By definition, if $\Gamma$ is a $\Phi_{d}$-complex, then the inclusion map $\alpha: \sigma \rightarrow \Gamma$ is $\mathbb{Z}_{2}$-equivariant, so the associated map $\widehat{\alpha}: \operatorname{Hom}(\Gamma, K) \rightarrow \operatorname{Hom}(\sigma, K)$ is also $\mathbb{Z}_{2}$ equivariant for each complex $K$.

Example 4.20 The graph $C_{2 r+1}$ is obviously an example of a $\Phi_{1}$-complex. Figure 5 displays four examples of $\Phi_{2}$-complexes, initial elements of two infinite series $\nabla_{\mu}$ and $\Sigma_{v}, \mu, v \in \mathbb{N}$. The complexes $\nabla_{1}$ and $\nabla_{2}$ etc. are obtained from two triangulated annuli, glued together along a common triangle $\sigma$. Similarly, the complexes $\Sigma_{1}, \Sigma_{2}, \ldots$, are obtained by gluing together two triangulated Möbius strips. The associated group of projectivities are $\Pi\left(\nabla_{\mu}, \sigma\right)=S_{3}$ and $\Pi\left(\Sigma_{v}, \sigma\right)=\mathbb{Z}_{2}$.

Theorem 4.21 Suppose that $\Gamma$ is a $\Phi_{d}$-complex in the sense of Definition 4.18, with an associated invariant simplex $\sigma=\left\{v_{0}, v_{1}, \ldots, v_{d}\right\}$. Suppose that $K$ is a pure

Fig. 5 Examples of

$\Phi_{2}$-complexes
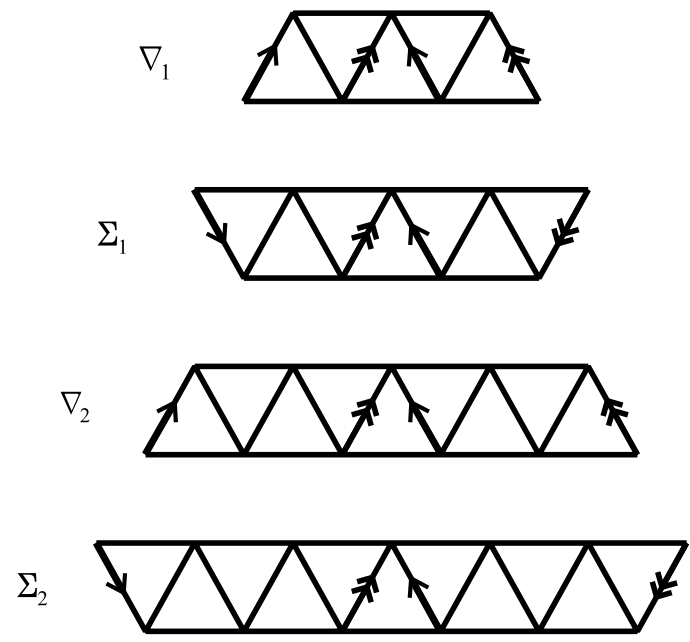
$d$-dimensional simplicial complex. Then for $m$ even,

$$
\operatorname{Coind}_{\mathbb{Z}_{2}}(\operatorname{Hom}(\Gamma, K)) \geq m \Rightarrow \chi(K) \geq m+d+2 .
$$

Proof By definition $\operatorname{Coind}_{\mathbb{Z}_{2}}(\operatorname{Hom}(\Gamma, K)) \geq m$ means that there exists a $\mathbb{Z}_{2}$-equivariant map $\mu: S^{m} \rightarrow \operatorname{Hom}(\Gamma, K)$. Assume that $\chi(K) \leq m+d+1$ which means that there exists a nondegenerate simplicial map $\phi: K \rightarrow \Delta^{[m+d+1]}$. By functoriality of the construction of Hom-complexes, Sect. 4.4.2, there is an induced $\mathbb{Z}_{2 \text { - }}$ equivariant map $\widehat{\phi}: \operatorname{Hom}(\Gamma, K) \rightarrow \operatorname{Hom}\left(\Gamma, \Delta^{[m+d+1]}\right)$ and similarly a map $\widehat{\alpha}$ : $\operatorname{Hom}\left(\Gamma, \Delta^{[m+d+1]}\right) \rightarrow \operatorname{Hom}\left(\sigma, \Delta^{[m+d+1]}\right)$. By [33] Theorem 3.3.3, the complex

$$
\operatorname{Hom}\left(\sigma, \Delta^{[m+d+1]}\right) \cong \operatorname{Hom}\left(K_{d+1}, K_{m+d+1}\right)
$$

is a wedge of $m$-dimensional spheres. Since $\operatorname{Hom}\left(\sigma, \Delta^{[m+d+1]}\right)$ is a free $\mathbb{Z}_{2}$-complex, we deduce that there exists a $\mathbb{Z}_{2}$-map $\operatorname{Hom}\left(\sigma, \Delta^{[m+d+1]}\right) \rightarrow S^{m}$. All these maps can be arranged in the following sequence of $\mathbb{Z}_{2}$-equivariant maps

$$
S^{m} \stackrel{\mu}{\longrightarrow} \operatorname{Hom}(\Gamma, K) \stackrel{\widehat{\phi}}{\longrightarrow} \operatorname{Hom}\left(\Gamma, \Delta^{[m+d+1]}\right) \stackrel{\widehat{\alpha}}{\longrightarrow} \operatorname{Hom}\left(\sigma, \Delta^{[m+d+1]}\right) \stackrel{v}{\longrightarrow} S^{m} .
$$

By Corollary 4.17, there is a homotopy equivalence $\widehat{\alpha} \simeq \tau \circ \widehat{\alpha}$. This contradicts Proposition 4.22, which completes the proof of the theorem.

Proposition 4.22 Suppose that $f: X \rightarrow Y$ is a $\mathbb{Z}_{2}$-equivariant map of free $\mathbb{Z}_{2}$ complexes $X$ and $Y$ where $\mathbb{Z}_{2}=\{1, \omega\}$. Assume that $\operatorname{Coind}_{\mathbb{Z}_{2}}(X) \geq m \geq \operatorname{Ind}_{\mathbb{Z}_{2}}(Y)$, where $m$ is an even integer. In other words our assumption is that there exist $\mathbb{Z}_{2}$ equivariant maps $\mu$ and $v$ such that

$$
S^{m} \stackrel{\mu}{\longrightarrow} X \stackrel{f}{\longrightarrow} Y \stackrel{v}{\longrightarrow} S^{m} .
$$

Then the maps $f$ and $\omega \circ f$ are not homotopic.

Proof If $f \simeq \omega \circ f: X \rightarrow Y$, then $v \circ f \circ \mu \simeq \nu \circ \omega \circ f \circ \mu: S^{m} \rightarrow S^{m}$ and by the equivariance of $v, \omega \circ g \simeq g: S^{m} \rightarrow S^{m}$ where $g:=v \circ f \circ \mu$. It follows that

$$
-\operatorname{deg}(g)=\operatorname{deg}(\omega) \operatorname{deg}(g)=\operatorname{deg}(\omega \circ g)=\operatorname{deg}(g),
$$

i.e. $\operatorname{deg}(g)=0$, which is in contradiction with a well-known fact [37] that a $\mathbb{Z}_{2}$ equivariant map $g: S^{m} \rightarrow S^{m}$ of spheres must have an odd degree.

Corollary 4.23 Suppose that $\Gamma$ is a $\Phi_{d}$-complex with an associated invariant simplex $\sigma=\left\{v_{0}, v_{1}, \ldots, v_{d}\right\}$. Suppose that $K$ is a pure $d$-dimensional simplicial complex. Then for $k$ odd,

$$
\operatorname{Hom}(\Gamma, K) \text { is } k \text {-connected } \Rightarrow \chi(K) \geq k+d+3 \text {. }
$$

Proof If $\operatorname{Hom}(\Gamma, K)$ is $k$-connected then $\operatorname{Coind}_{\mathbb{Z}_{2}}(\operatorname{Hom}(\Gamma, K)) \geq k+1$, hence the implication (17) is an immediate consequence of Theorem 4.21. 


\subsection{Flag Complexes and the Homotopy Test Graphs}

A simplicial complex is a flag-complex if $\sigma \in K$ if and only if the associated 1 -skeleton $\sigma^{(1)}$ of $\sigma$ is a subcomplex of $K$. Examples of flag-complexes include the clique-complex $C$ lique $(G)$ of a graph and the order complex $\Delta(P)$ of all chains (flags) in a poset $P$.

If $K$ is a flag-complex and $G=G_{K}:=K^{(1)}$ is the associated vertex-edge graph of $K$, then $K=$ Clique $(G)$. From here we deduce, relying on the isomorphism given in Example 4.8, that if $K$ and $L$ are flag complexes, then

$$
\operatorname{Hom}(K, L) \cong \operatorname{Hom}\left(G_{K}, G_{L}\right) \text {. }
$$

This simple observation allows us to transfer results about simplicial complexes back to graphs. An example is the following theorem which can be deduced from Corollary 4.23 .

Theorem 4.24 Suppose that $W$ is $\Phi_{d}$-complex (Definition 4.18) which is also a flag complex. Assume that $k$ is an odd integer. Let $T:=W^{(1)}$. Then for each graph $G$

$$
\operatorname{Hom}(T, G) \text { is } k \text {-connected } \Rightarrow \chi(G) \geq k+d+3 \text {. }
$$

Examples of $\Phi_{2}$-complexes $W$ satisfying conditions of Theorem 4.24 are the complexes $\Sigma_{1}, \nabla_{2}, \Sigma_{2}$ depicted in Fig. 5. Moreover, for all associated graphs $\chi\left(W^{(1)}\right)=4$ which in light of (19) means that they are all candidates for "homotopy test graphs" in the sense of [34, Chap. 6].

\section{Subsequent Developments}

In this section we briefly outline some of the main developments after the appearance of preprints [49] and [50], where the original approach to combinatorial groupoids was developed and which were eventually subsumed by this paper.

The review paper [51] is a leisurely introduction to combinatorial groupoids, as envisaged by [50] and this paper, with numerous examples which range from "Penrose tribar" to the Lloyd "15 game". [51] also gives pointers to different but related developments involving "discrete connections", "parallel transport on discrete structures", discretization of curvature etc. For example, it includes references and a brief description of the work of Bolker, Guillemin, and Holm (loc. cit.) on connections on graphs which was motivated by the theory of $G K M$-manifolds (named after Goresky, Kottwitz, and MacPherson). Bolker et al., guided by an analogy between graph theory and the theory of manifolds, developed a theory which fits well into the general scheme outlined in Sect. 2 (see also Sect. 4.2).

Perhaps the most interesting subsequent new development is related to Sect. 4 (Lovász Hom-conjecture and its ramifications). The original proof [4] of Lovász Hom-conjecture was quite long and technically involved, partly because it develops a broader framework for studying homology of Hom-complexes. For illustration, a more detailed presentation of the proof with some background material required 
a whole volume of the IAS/Park City Mathematics Series [34]. The short, elementary proof presented here in Sect. 4.3 (see also the announcement [49]), covering the Lovász conjecture for the even number of colors, was the first indication that much shorter and less-involved proofs are possible. The first complete, short proof of Lovász Hom-conjecture was subsequently found by Schultz [44], see also [45] for a detailed comparison of different topological bounds for chromatic numbers and analysis of homotopy test graphs (cf. Sect. 4.6). Finally Kozlov in [35] established an even more transparent and elementary proof based on a short and elegant calculation of the height of the first Stiefel-Whitney class of the $\mathbb{Z}_{2}$-space $\operatorname{Hom}\left(C_{2 r+1}, K_{n}\right)$.

\section{References}

1. Babson, E., Barcelo, H., De Longueville, M., Laubenbacher, R.: Homotopy theory for graphs. J. Algebr. Comb. 24(1), 31-44 (2006). arXiv:math.CO/0403146 v1, 9 March 2004

2. Babson, E.K., Chan, C.: Counting faces for cubical spheres modulo two. Discrete Math. 212, 169-183 (2000)

3. Babson, E., Kozlov, D.N.: Complexes of graph homomorphisms. Isr. J. Math. 152, 285-312 (2006). MR2214465 (2007b:52024), arXiv:math.CO/0310056 v1, 5 October 2003

4. Babson, E., Kozlov, D.N.: Proof of the Lovász conjecture. Ann. Math. 165(3), 965-1007 (2007). arXiv:math.CO/0402395 v2, 2004

5. Barcelo, H., Laubenbacher, R.: Perspectives on $A$-homotopy theory and its applications. Discrete Math. 298, 39-61 (2005)

6. Barcelo, H., Kramer, X., Laubenbacher, R., Weaver, C.: Foundations of a connectivity theory for simplicial complexes. Adv. Appl. Math. 26, 97-128 (2001)

7. Bern, M.W., Eppstein, D., Erikson, J.G.: Flipping cubical meshes. Eng. Comput. 18, 173-187 (2002)

8. Björner, A.: Topological methods. In: Graham, R., Grötschel, M., Lovász, L. (eds.) Handbook of Combinatorics. North-Holland, Amsterdam (1995)

9. Björner, A., Las Vergnas, M., Sturmfels, B., White, N., Ziegler, G.: Oriented Matroids. Encyclopedia of Mathematics and its Applications, vol. 46. Cambridge University Press, Cambridge (1993)

10. Bolker, E.D., Guillemin, V.W., Holm, T.S.: How is graph like a manifold? arXiv:math.CO/0206103 v1 (2002)

11. Brown, R.: Topology and Groupoids. Booksurge LLC (2006); retitled, revised, updated and extended edition of Topology: A Geometric Account of General Topology, Homotopy Types and the Fundamental Groupoid. Ellis Horwood, Chichester (1988), and of Elements of Modern Topology. McGraw-Hill, New York (1968)

12. Brown, R.: From groups to groupoids: a brief survey. Bull. Lond. Math. Soc. 19, 113-134 (1987)

13. Buchstaber, V.M., Panov, T.E.: Torus actions and their applications in topology and combinatorics. In: Univ. Lecture Ser., vol. 24. AMS, Providence (2002)

14. Conner, P.E., Floyd, E.E.: Fixed point free involutions and equivariant maps. Bull. Am. Math. Soc. 66, 416-441 (1960)

15. Connes, A.: Non Commutative Geometry. Academic Press, San Diego (1995)

16. Dynnikov, I.A., Novikov, S.P.: Geometry of the triangle equation on two-manifolds. Moscow Math. J. 3(2), 419-438 (2003).

17. Dolbilin, N.P., Shtan'ko, M.A., Shtogrin, M.I.: Cubic subcomplexes in regular lattices. Dokl. Akad. Nauk. SSSR 291, 277-279 (1986). English translation: Sov. Math. Dokl. 34 (1987)

18. Dolbilin, N.P., Shtan'ko, M.A., Shtogrin, M.I.: Cubic manifolds in lattices. Izv. Ross. Akad. Nauk, Ser. Mat. 58, 93-107 (1994). English translation: Russ. Acad. Sci. Izv. Math. 44, 301-313 (1995)

19. Eppstein, D.: Linear complexity hexaedral mesh generation. Comput. Geom. 12, 3-16 (1999)

20. Funar, L.: Cubulations, immersions, mappability and a problem of Habegger. Ann. Sci. Ec. Norm. Super. 32, 681-700 (1999) 
21. Funar, L.: Cubulations mod bubble moves. In: Nencka, H. (ed.) Proc. Conf. Low Dimensional Topology, Funchal, Madeira 1998. Contemporary Math., vol. 233, pp. 29-43. AMS, Providence (1999)

22. Funar, L.: Surface cubications mod flips. Manuscr. Math. 125, 285-307 (2008). arXiv:math.GT/ 0501550, September 2005

23. Godsil, C., Royle, G.: Algebraic Graph Theory. Graduate Texts in Mathematics, vol. 207. Springer, Berlin (2001)

24. Higgins, P.J.: Categories and Groupoids. Van Nostrand-Reinhold, London (1971)

25. Izmestiev, I.: 3-dimensional manifolds defined by simple polytopes with colored facets. Mat. Zametki 69, 375-382 (2001). English translation: Math. Notes 69 (1987)

26. Izmestiev, I.: Free actions of a torus on a manifold $\mathcal{L}_{P}$ and the projectivity group of a polytope $P$. Usp. Mat. Nauk 56, 169-170 (2001). English translation: Russ. Math. Surv. 56 (2001)

27. Izmestiev, I., Joswig, M.: Branched coverings, triangulations, and 3-manifolds. Adv. Geom. 3, 191225 (2003). arXiv:math.GT/0108202 v2, 20 March 2002

28. Joswig, M.: Projectives in simplicial complexes and coloring of simple polytopes. Math. Z. 240(2), 243-259 (2002). arXiv:math.CO/186 v3, 27 June 2001

29. Joswig, M.: Projectives in simplicial complexes and coloring of simple polytopes (extended abstract). Russ. Math. Surv. 56, 584-585 (2001)

30. Karalashvili, O.R.: On mappings of cubic manifolds into the standard lattice of Euclidean space. Tr. Mat. Inst. Steklov 196, 86-89 (1991). Translated in: Proc. Steklov Inst. Math. 196 (1992)

31. Kirby, R.: Problems in low-dimensional topology. In: Kazez, W.H. (ed.) Geometric Topology, Georgia International Topology Conference. AMS-IP Studies in Advanced Math. vol. 2, pp. 35-473, part 2 (1995)

32. Kozlov, D.N.: Complexes of directed trees. J. Comb. Theory Ser. A 88(1), 112-122 (1999)

33. Kozlov, D.N.: A simple proof for folds on both sides in complexes of graph homomorphisms. Proc. Am. Math. Soc. (2004, to appear). arXiv:math.CO/0408262 v2, December 2004

34. Kozlov, D.N.: Chromatic numbers, morphism complexes, and Stiefel-Whitney characteristic classes. In: Geometric Combinatorics. IAS/Park City Mathematics Series, vol. 14. American Mathematical Society, Providence (2008, in press). Institute for Advanced Study (IAS), Princeton, NJ

35. Kozlov, D.N.: Cobounding odd cycle colorings. Electron. Res. Announc. Am. Math. Soc. 12, 53-55 (2006). arXiv.math/0602561v1 [math.AT] 24, February 2006

36. Lovász, L.: Kneser's conjecture, chromatic number and homotopy. J. Comb. Theory Ser. A 25, 319324 (1978)

37. Matoušek, J.: Using the Borsuk-Ulam Theorem. Lectures on Topological Methods in Combinatorics and Geometry. Springer, Berlin (2003)

38. Matoušek, J., Ziegler, G.: Topological lower bounds for the chromatic number; A hierarchy. Jahresber. DMV (2008, to appear). arXiv:math.CO/0208072 v3, 24 November 2003

39. Novikov, S.P.: Topology I. Encyclopaedia Math. Sci., vol. 12. Springer, Berlin (1996)

40. Novikov, S.P.: Discrete connections and difference linear equations. Tr. MIAN 247, 186-201 (2004)

41. Quillen, D.: Homotopy properties of the poset of nontrivial $p$-subgroups of a group. Adv. Math. 28, 101-128 (1978)

42. Segal, G.: Classifying spaces and spectral sequences. Publ. Math. I.H.E.S. 34, 105-112 (1968)

43. Schwartz, A., Ziegler, G.M.: Construction techniques for cubical complexes, odd cubical 4-polytopes, and prescribed dual manifolds. Exp. Math. 13, 385-413 (2004). arXiv:math.CO/0310269 v3, 2 January 2004

44. Schultz, C.: A short proof of $w_{1}^{n}\left(\operatorname{Hom}\left(C_{2 r+1} K_{n+2}\right)\right)=0$ for all $n$ and a graph colouring theorem of Babson and Kozlov. Isr. J. Math. (2008, to appear). arXiv:math.AT/0507346 v2 (August 2005)

45. Schultz, C.: Graph colourings, spaces of edges, and spaces of circuits. arXiv:math/0606763v1 [math.CO] (29 June 2006)

46. Weinstein, A.: Groupoids: Unifying internal and external symmetry. Not. Am. Math. Soc. 43, 744-752 (1996)

47. Ziegler, G.M.: Lectures on Polytopes, 2nd edn. Graduate Texts in Mathematics, vol. 152. Springer, Berlin (1998)

48. Živaljević, R.T.: $W I$-posets, graph complexes and $\mathbb{Z}_{2}$-equivalences. J. Comb. Theory Ser. A 111, 204-223 (2005)

49. Živaljević, R.: Parallel transport of Hom-complexes and the Lovász conjecture. arXiv:math.CO/0506075 v1 (3 June 2005)

50. Živaljević, R.: Combinatorial groupoids, cubical complexes, and the Lovász conjecture. arXiv:math.CO/0510204 v2 (October 2005)

51. Živaljević, R.: Groupoids in combinatorics-applications of a theory of local symmetries. Proc. Conference "Algebraic and Geometric Combinatorics", Anogia, Crete 2005. Contemporary Mathematics, vol. 423, pp. 305-324 